\title{
Tendencias en las evaluaciones de riesgo al colapso de ecosistemas terrestres y humedales
}

\section{Trends in collapse risk assessments of terrestrial and wetland ecosystems}

\author{
Carlos A. Masés-García', Bernal Herrera-Fernández,2,3 y Miguel Briones-Salas ${ }^{*}$ \\ 1 Instituto Politécnico Nacional. CIIDIR. Unidad \\ Oaxaca. Santa Cruz Xoxocotlán, Oaxaca, México. \\ cmasesg@gmail.com, bernal.herrera.f(a)gmail.com \\ 3 Universidad Nacional. Instituto Internacional para el * Autor de correspondencia. mbriones@ipn.mx \\ Manejo de la Vida Silvestre. Costa Rica. \\ 2 Unión Internacional para la Conservación de la \\ Naturaleza. Comisión sobre el Manejo de \\ Ecosistemas. Gland, Suiza.
}

\section{RESUMEN}

Las actividades antropogénicas amenazan cada vez más la extensión y procesos de los ecosistemas. La Lista Roja de Ecosistemas (LRE) de la Unión Internacional para la Conservación de la Naturaleza (IUCN, por sus siglas en inglés) es un estándar global para evaluar su riesgo de colapso. En este trabajo se analizaron las tendencias mundiales en las evaluaciones de riesgo de los ecosistemas terrestres y humedales, mediante la búsqueda y análisis de publicaciones del 2010 a 2019 que aplicaron los protocolos de la LRE. Se encontraron 43 publicaciones que evaluaron 1227 ecosistemas: bosques (531), matorrales (181), pastizales y herbazales (212), humedales (166), costeros (42) y de vegetación escasa o poco comunes (95). Se registraron 15 criterios para delimitar los ecosistemas, destacó el tipo de vegetación usada como proxy en todos los casos. Existió una fuerte variación en las extensiones de los ecosistemas. Se identificaron 34 variables para definir umbrales de colapso: una espacial, 15 bióticas y 18 abióticas; el cambio en la cobertura vegetal, extensión y distribución geográfica fueron de aplicación general. Los periodos de datos fiables para evaluar ecosistemas van de 12 a 48 años, con ellos se infirieron lapsos de 50 años (pasado o futuro), o se dedujeron cambios desde 1750. Sobresalieron los ecosistemas en "Preocupación Menor" (412), se encontraron solo dos "colapsados" y no hubo diferencia significativa entre amenazados y no-amenazados. Las evaluaciones analizadas incidieron en 69 países, se realizaron a escalas continentales, nacionales y subnacionales. Se proponen mejoras a los protocolos de la LRE y se proporciona información clave para aplicar en evaluaciones de riesgo ecosistémico en el futuro.

PALABRAS CLAVE: ecosistemas amenazados, ecosistemas en peligro, evaluación ecosistémica, Lista Roja de Ecosistemas de la IUCN, política de conservación, práctica de conservación.

\section{ABSTRACT}

Anthropogenic activities threaten more and more the extension and processes of ecosystems. The IUCN Red List of Ecosystems (RLE) is a global standard for assessing their risk of collapse. In this paper, global trends of risk assessments of terrestrial and wetland ecosystems were analyzed through a search for and analysis of publications from 2010 to 2019 that utilized the RLE protocols. Forty-three publications that evaluated 1227 ecosystems: forests (531), scrublands (181), grasslands and herbaceous (212), wetlands (166), coastal (42), and sparsely vegetated or uncommon ecosystems (95) were found. Fifteen criteria were recorded to delimit the ecosystems, highlighting the type of vegetation used as a proxy in all cases. There was a strong variation in the extension of the ecosystems evaluated. Thirty-four variables were identified to define collapse thresholds: one spatial, 15 biotics, and 18 abiotics; vegetation cover change, geographic distribution, and extension were variables of general application. The periods of reliable data for assessing ecosystems range from 12 to 48 years; with them, 50-year periods (past or future) or changes deductied since 1750 were inferred. Ecosystems in "Least Concern" (412) stood out, only two "collapsed" were found, and there was no significant difference between threatened and nonthreatened. The assessments analyzed comprised 69 countries, which were conducted at continental, national, and subnational scales. Improvements in the RLE protocols are proposed and key information is provided for the application of ecosystems risk assessment in the future.

KEYWORDS: threatened ecosystems, endangered ecosystems, ecosystem assessment, IUCN Red List of Ecosystems, conservation policy, conservation practice. 


\section{INTRODUCCIÓN}

Actualmente, los ecosistemas terrestres han disminuido en promedio $47 \%$ de su estado natural inicial y los humedales han perdido más de $85 \%$ de su extensión, se estima que $75 \%$ de ecosistemas de tierra firme están impactados por actividades antropogénicas (Plataforma Intergubernamental Científico-Normativa Sobre Biodiversidad y Servicios de los Ecosistemas [Ipbes], 2019). La medición de estos cambios y sus amenazas son esenciales para la toma de decisiones, el diseño de políticas y estrategias de conservación (Nicholson et al., 2012; Rowland et al., 2018). Para ello, en 2008 surgió la Lista Roja de Ecosistemas de la IUCN, un estándar global que ha evaluado y clasificado a los ecosistemas de acuerdo con su riesgo de colapso (Bland, Keith, Miller, Murray y Rodríguez, 2017a). Sin embargo, hasta hoy no se conocen las tendencias de las evaluaciones de riesgo aplicadas a ecosistemas terrestres y humedales.

A partir de la Primera Cumbre de la Tierra en 1972 creció el interés por conocer el estado de los ecosistemas, se desarrollaron cientos de investigaciones sobre sus funciones y servicios (Cardinale et al., 2012). Esto condujo a la Evaluación de Ecosistemas del Milenio realizada en el 2001, una de sus sugerencias fue diseñar métodos de evaluación de riesgo para estimar sus amenazas (Hassan, Scholes y Ash, 2005; Carpenter et al., 2006). Algunos países desarrollaron y aplicaron sus protocolos para evaluar ecosistemas en su jurisdicción (Nicholson et al., 2015; Bland et al., 2017b); sin embargo, exhibieron inconsistencias en la presentación de informes ambientales entre países, dificultando la comparación de resultados (Nicholson et al., 2015).

$\mathrm{El}$ análisis de riesgo requiere un enfoque estandarizado para la delimitación de los ecosistemas, criterios y umbrales en los estados de amenaza (Rodríguez et al., 2011). Por ello, en el Cuarto Congreso Mundial de la Naturaleza de la IUCN (2008) se planteó la iniciativa de la LRE (Nicholson, Keith y Wilcove, 2009; Rodríguez et al., 2011), y posteriormente se desarrolló su estándar global para evaluar el riesgo de los ecosistemas (Keith et al. 2015). En el 2013, Keith et al. (2013b) establecieron los fundamentos científicos de la LRE y presentaron una compilación de estudios realizados en el mundo donde la aplicaron. Estos autores por primera vez introdujeron el término "colapso" en la LRE para definir el punto final del ecosistema: su desaparición total o su transformación en uno distinto o novel. En 2014 la IUCN adoptó a la LRE como una iniciativa global y en el 2016 se publicó la primera versión de las Directrices para la Aplicación de sus Categorías y Criterios que se actualizó en 2017 a la versión vigente (Bland, Keith, Miller, Murray y Rodríguez, 2016; Bland et al., 2017a).

La LRE establece cinco criterios para evaluar el riesgo de colapso: A) reducción en la distribución del ecosistema; B) distribución restringida relacionada a una reducción o amenaza persistente y explícita; C) degradación ambiental (abiótico), D) alteración de los procesos bióticos y E) la modelación matemática del colapso. Los criterios A, C y D se analizan en periodos de 50 años al: pasado (subcriterios A1, C1, D1), presente-pasado (A2b, C2b y D2b) y futuro (A2a, C2a y D2a), o bien un periodo histórico a partir de 1750 (A3, C3 y D3) (Keith et al., 2013b; Bland et al., 2017a). Los resultados se acotan a categorías de riesgo de acuerdo con umbrales establecidos: colapsado (CO), en peligro crítico (CR), en peligro (EN), vulnerable (VU), casi amenazado (NT), preocupación menor (LC), datos insuficientes (DD) y no evaluado (NE) (Bland et al., 2016; Bland et al., 2017a). Estas se agrupan en dos rubros: ecosistemas no-amenazados que incluyen las categorías LC y NT, y ecosistemas amenazados con las categorías VU, EN y CR (Bland et al., 2017a).

La LRE es una herramienta útil para priorizar áreas de conservación y restauración, reconocer presiones y amenazas (Janssen et al., 2016; Ferrer-Paris et al., 2019), abordar vacíos de representatividad de ecosistemas que no están en áreas protegidas e informar el estado de riesgo para que los gobiernos implementen marcos legislativos, planificación del territorio y gestión de ecosistemas (Rodríguez, Rojas-Suarez y Giraldo-Hernández, 2010; Herrera-F, Zamora y Chacón, 2015; Bland et al., 2019). Asimismo, apoya en el monitoreo del cumplimiento de objetivos globales sobre biodiversidad que requieren la 
presentación de informes sobre cambios de los ecosistemas, es de aplicación potencial en las Metas de Aichi, la Convención de Ramsar, los sitios de Patrimonio Mundial de la Organización de las Naciones Unidas para la Educación, la Ciencia y la Cultura (Unesco, por sus siglas en inglés) y la Ipbes (Nicholson et al., 2015).

Los Acuerdos del Congreso Mundial de la Naturaleza de la IUCN en 2012 establecen como meta que para el 2025 se realice la evaluación de los ecosistemas del mundo con el protocolo de la LRE (Bland et al., 2016). No obstante, para ver sus avances es clave conocer las tendencias de las evaluaciones de riesgo en ecosistemas terrestres $\mathrm{y}$ humedales: ¿Cuántas publicaciones se han realizado, con qué características y frecuencia? ¿Qué tipo de evaluaciones y en qué dimensión territorial se han aplicado? ¿Qué ecosistemas se han analizado y cuáles elementos de delimitación y tamaño se han empleado? ¿Qué variables, criterios de la LRE y marcos temporales se han empleado para determinar el "colapso"? ¿Cuál es el estado de riesgo global de los ecosistemas? y ¿Cuáles son los avances en las diferentes regiones del planeta? Las respuestas a estas preguntas serán cruciales para retroalimentar nuevas evaluaciones, informar de las tendencias para la toma de decisiones relacionadas a la LRE y determinar necesidades de investigación a desarrollar en nuevos estudios.

\section{OBjetivos}

Analizar las tendencias globales en las evaluaciones de riesgo de colapso de los ecosistemas terrestres y humedales que utilizan los protocolos de la LRE con base en: 1) las características de las publicaciones y su frecuencia temporal, 2) los tipos de evaluaciones realizadas y su dimensión territorial, 3) los tipos de ecosistemas, extensión, tamaños y criterios de delimitación, 4) las variables utilizadas para evaluar el colapso, la frecuencia con que se utilizaron los criterios de la LRE y los marcos temporales de datos fiables empleados, 5) los estados de riesgo de los ecosistemas y 6) la distribución geográfica de las publicaciones y ecosistemas evaluados.

\section{MATERIALES Y MÉTODOS}

\section{Obtención de información}

Se realizó una búsqueda en motores de información científica: Scopus, Web of Science, Science Direct, Scholar Google y Conricyt (Consorcio Nacional de Información Científica y Tecnológica de México), entre los años 2010 y 2019 (no se restringió el periodo de búsqueda, el resultado más antiguó más antiguo data del 2010), usando los términos "Red List of Ecosystem", "IUCN ecosystem assessment", "endangered ecosystem", "ecosystem collapse" y "ecosystem assessment". Se restringieron los resultados a aquellos estudios que realizaron evaluaciones de riesgo de colapso bajo los protocolos de la LRE en ecosistemas terrestres y humedales, se excluyeron ecosistemas marinos y dulceacuícolas.

\section{Análisis}

Se clasificaron y analizaron las publicaciones por: 1) tipo de documento (artículos científicos, libros e informes técnicos/reportes), editorial y año; 2) clase de evaluaciones realizadas: "regionales" donde se evalúan partes de ecosistemas a nivel continental, nacional o subnacional; "globales" que analizan sus extensiones totales (IUCNCommission on Ecosystem Management [CEM], 2016; Bland et al., 2017a); "estratégicas" que son detalladas, dirigidas a uno o pocos tipos de ecosistemas, y "sistemáticas" donde se analizan un gran número dentro de un área determinada (Bland et al., 2019).

Los ecosistemas registrados se agruparon de acuerdo con sus formaciones vegetales dominantes (fisonomía): bosques, matorrales, pastizales y herbazales, humedales, costeros (partes terrestres) y ecosistemas con vegetación escasa o poco comunes (Oliveira-Miranda et al., 2010; Janssen et al., 2016; Velazquez, Medina-García, DuránMedina, Amador y Gopar-Merino, 2016; Tan et al., 2017). Se determinó la frecuencia anual con que fueron evaluados y se analizaron los datos de sus áreas mediante estadísticos 
descriptivos $(\bar{X}, \mathrm{~S}, \mathrm{Me})$. Asimismo, se establecieron las variables utilizadas para la delimitación de unidades de evaluación y su frecuencia de aplicación, de acuerdo con sus características bióticas, factores ambientales, distribución, procesos y límites administrativos (Bland et al., 2018; Rowland et al., 2018).

Con base en el método de Bland et al. (2018) y Rowland et al. (2018), se determinaron las variables empleadas en cada estudio para definir el colapso, se agruparon de acuerdo con indicadores bióticos, abióticos y espaciales. Se comparó la frecuencia de cada variable y los criterios y subcriterios de la LRE donde se emplearon. Asimismo, se determinaron los periodos temporales utilizados: 50 años al pasado (subcriterios A1, C1 y D1), 50 entre pasadopresente-futuro (A2b, C2b y D2b) y 50 a futuro (A2a, C2a y D2a), o bien un periodo histórico desde 1750 (A3, C3, B3) (Bland, Keith, et al., 2017; Keith et al., 2013a).

Los ecosistemas se agruparon de acuerdo con sus categorías de riesgo: CO, CR, EN, VU, NT y LC, y se determinó la proporción global de cada una. Se consideró el estado de riesgo más alto de cada ecosistema, no se tomaron en cuenta las categorías Datos Deficientes (DD) y No Evaluado (NE) por no dar información del estado de riesgo. Posteriormente se compararon estadísticamente mediante una prueba no-paramétrica Wilcoxon (U MannWhitney) los "ecosistemas amenazados" (CR, EN y VU) y "no amenazados" (NT y LC), tomando como una observación el número de ecosistemas registrados en cada categoría por publicación. Finalmente, se determinó la frecuencia en el número de publicaciones generadas en cada país y el número de ecosistemas evaluados; mediante un sistema de información geográfica se proyectaron espacialmente.

\section{Resultados}

La búsqueda arrojó 1598 documentos, de ellos el análisis se acotó a 43 publicaciones que evaluaron 1227 ecosistemas terrestres y humedales bajo los protocolos de la LRE (Tabla 1). Corresponden a 30 artículos de revistas en el Journal Citation Report (JCR) (69.77\%), cinco libros (11.63\%) y ocho informes o reportes (18.60\%). Se publicaron en 13 editoriales, ocho de ellas únicamente tienen una publicación, 55.81\% se concentró en Plos One (15) y Austral Ecology (9) (Fig. 1). Hubo un promedio anual de 4.3 publicaciones, destacaron el 2015 (14) y 2013(13) (Fig. 2). Siete publicaciones fueron evaluaciones de tipo "global/ estratégicas" que abarcan $0.57 \%$ de los 1227 ecosistemas; 36 documentos correspondieron a evaluaciones "regionales": 16 "estratégicas" y 20 "sistemáticas" que suman 1.30\% y $98.13 \%$ de los ecosistemas, respectivamente (Tabla 1). Veinticuatro publicaciones indicaron el análisis de un solo ecosistema, el valor más alto fue de 206 con un promedio de 29.21 ecosistemas por publicación. De ellas destacan dos realizadas a nivel continental en América y Europa, y diez a escala nacional (Tabla 1).

Los ecosistemas registrados son: 531 bosques, 181 matorrales, 212 pastizales y herbazales, 166 humedales, 42 costeros y 95 con vegetación escasa o poco comunes (Tabla 1). El promedio anual fue de 122.7 ecosistemas; sin embargo, durante los últimos cinco años se realizó el análisis de 90.63\% (Fig. 2). Solo 274 ecosistemas indicaron su extensión con un valores entre $0.28 \mathrm{~km}^{2}$ y 311496.00 $\mathrm{km}^{2}\left(\bar{X}=9015.85 \mathrm{~km}^{2}, \mathrm{~S}=31204.16, \mathrm{Me}=712.40 \mathrm{~km}^{2}\right)$.

Se encontraron 15 criterios para la delimitación de ecosistemas, agrupados en 10 variables de tipo ambiental, tres relacionadas a divisiones administrativas, una biótica y una de proceso (Tabla 2). El tipo de vegetación (variable biótica) se utilizó en todos los estudios; la temperatura y precipitación fueron los dos principales factores ambientales empleados y las fronteras de los países fue el elemento administrativo más utilizado (Tabla 2).

Se registraron 34 variables aplicadas para definir el colapso: 18 abióticas, 15 bióticas y una espacial. El cambio en la cobertura vegetal, extensión y distribución geográfica se emplearon en todas las evaluaciones. La invasión de especies exóticas de flora y fauna, y los cambios en la distribución, riqueza y abundancia de especies claves modeladoras fueron las variables bióticas más representativas utilizadas en 21 y 16 casos, respectivamente. Mientras que las variables bioclimáticas en escenarios de cambio climático y los cambios hidrológicos fueron las más importantes de tipo abiótico utilizadas en 23 y 13 ocasiones, respectivamente (Tabla 3). 
TABLA 1. Publicaciones que evalúan el riesgo de colapso de ecosistemas terrestres y humedales bajo los protocolos de la Lista Roja de Ecosistemas de la Unión Internacional para la Conservación de la Naturaleza.

\begin{tabular}{|c|c|c|c|c|c|c|c|}
\hline Evaluación & Tipo de evaluación & Tipo de ecosistema & Referencia & $\begin{array}{l}\text { Criterios } \\
\text { aplicados }\end{array}$ & $\begin{array}{l}\text { No de } \\
\text { ecosis- } \\
\text { temas }\end{array}$ & $\begin{array}{l}\text { Categorías de } \\
\text { riesgo }\end{array}$ & Países \\
\hline $\begin{array}{l}\text { Bosques } \\
\text { templados y } \\
\text { tropicales de } \\
\text { América }\end{array}$ & $\begin{array}{l}\text { Regional/sistemática } \\
\text { Escala continental }\end{array}$ & 123 bosques & $\begin{array}{l}\text { (Ferrer-Paris } \\
\text { et al., 2019) }\end{array}$ & $\begin{array}{l}\mathrm{A} 1, \mathrm{~A} 2 \mathrm{~b}, \mathrm{~A} 3, \\
\mathrm{~B} 1, \mathrm{~B} 2, \mathrm{~B} 3, \\
\mathrm{C} 2 \mathrm{a}, \mathrm{C} 2 \mathrm{~b}, \mathrm{D} 1, \\
\mathrm{D} 2 \mathrm{~b}, \mathrm{D} 3\end{array}$ & 123 & $\begin{array}{l}13 \text { CR, } 48 \text { EN, } \\
37 \text { VU, } 12 \text { NT y } \\
13 \text { LC }\end{array}$ & $\begin{array}{l}21 \text { países del } \\
\text { continente } \\
\text { americano }\end{array}$ \\
\hline
\end{tabular}

31 humedales 19 pantanos y vegetación rivereña y 12 turberas y afloramientos de agua), 9 poco comunes

Lista Roja de Hábitats de la República

Regional/sistemática Checa

(acantilados, graveras y cuevas), 14 de vegetación escasa (hábitats alpinos sin árboles), 44 pastizales y herbazales, 7 matorrales y 37 bosques.

\begin{tabular}{|c|c|c|c|c|c|c|c|}
\hline $\begin{array}{l}\text { Hábitats } \\
\text { Finlandeses }\end{array}$ & $\begin{array}{l}\text { Regional/sistemática } \\
\text { Escala Nacional }\end{array}$ & $\begin{array}{l}61 \text { bosques, } 64 \text { herbazales } \\
\text { y pastizales, } 10 \text { matorrales, } \\
71 \text { humedales-pantanos }\end{array}$ & $\begin{array}{l}\text { (Kontula y } \\
\text { Raunio, 2018) }\end{array}$ & $\begin{array}{l}\text { A1, A2a, A2b, } \\
\text { A3, B1, B2, } \\
\text { B3, C1, C2a, } \\
\text { C2b, C3, D1, } \\
\text { D2a, D2b, D3 }\end{array}$ & 206 & $\begin{array}{l}53 \mathrm{CR}, 37 \mathrm{EN}, \\
52 \mathrm{VU}, 33 \mathrm{NT} \\
\text { y } 52 \mathrm{LC}\end{array}$ & Finlandia \\
\hline $\begin{array}{l}\text { Bosques } \\
\text { mediterráneos } \\
\text { de Francia }\end{array}$ & $\begin{array}{l}\text { Regional/sistemática } \\
\text { Escala Nacional }\end{array}$ & 14 bosques & $\begin{array}{l}\text { (UICN-France, } \\
\text { 2018) }\end{array}$ & $\begin{array}{l}\text { A1, A2a, A2b, } \\
\text { A3, B1, B2, } \\
\text { B3, C1, C2a, } \\
\text { C2b, C3, D1, } \\
\text { D2a, D2b, } \\
\text { D3, E }\end{array}$ & 14 & $\begin{array}{l}1 \mathrm{EN}, 3 \text { VU, } 7 \\
\text { NT, 3 VU. }\end{array}$ & Francia \\
\hline $\begin{array}{l}\text { Manglar, } \\
\text { Filipinas }\end{array}$ & Regional/sistemática & Humedal-manglar & $\begin{array}{l}\text { (Marshall et } \\
\text { al., 2018) }\end{array}$ & $\begin{array}{l}\text { A2b, B1, B2, } \\
\text { B3 }\end{array}$ & 1 & LC & Filipinas \\
\hline $\begin{array}{l}\text { Suroeste de } \\
\text { China }\end{array}$ & $\begin{array}{l}\text { Regional/sistemática } \\
\text { Escala Nacional y } \\
\text { subnacional }\end{array}$ & $\begin{array}{l}43 \text { bosques, } 36 \text { matorrales, } \\
2 \text { humedales, } 24 \text { pastizales. }\end{array}$ & $\begin{array}{l}\text { (Tan et al., } \\
\text { 2017) }\end{array}$ & $\begin{array}{l}\mathrm{A} 1, \mathrm{~A} 2 \mathrm{~b}, \mathrm{B1}, \\
\mathrm{B} 2\end{array}$ & 105 & $\begin{array}{l}9 \text { CR, } 14 \text { EN, } 12 \\
\text { VU, } 15 \text { NT y } 55 \\
\text { LC. }\end{array}$ & China \\
\hline $\begin{array}{l}\text { Ecosistemas } \\
\text { de Colombia } \\
\text { (dos } \\
\text { publicaciones: } \\
\text { una } \\
\text { actualizada) }\end{array}$ & $\begin{array}{l}\text { Regional/sistemática } \\
\text { Escala Nacional }\end{array}$ & $\begin{array}{l}54 \text { bosques, } 22 \text { matorrales } \\
\text { (páramos) y } 5 \text { herbazales. }\end{array}$ & $\begin{array}{l}\text { (Etter, } \\
\text { Andrade, } \\
\text { Amaya y } \\
\text { Arévalo, } \\
\text { 2015), } \\
\text { Actualización } \\
\text { en } 2017 \\
\text { (Etter et al., } \\
\text { 2017) }\end{array}$ & $\begin{array}{l}A 1, A 2 a, A 2 b \\
A 3\end{array}$ & 81 & $\begin{array}{l}20 \mathrm{CR}, 18 \mathrm{EN} \\
15 \mathrm{VU}, 28 \mathrm{LC}\end{array}$ & Colombia \\
\hline $\begin{array}{l}\text { Lista Roja de } \\
\text { Hábitats } \\
\text { terrestres y } \\
\text { dulceacuícola } \\
\text { s de Europa. }\end{array}$ & $\begin{array}{l}\text { Regional/sistemática } \\
\text { Escala continental }\end{array}$ & $\begin{array}{l}29 \text { costeros, } 13 \text { humedales } \\
\text { (turberas y pantanos), } 53 \\
\text { pastizales, } 37 \text { matorrales, } 41 \\
\text { bosques y } 25 \text { vegetación } \\
\text { escasa. }\end{array}$ & $\begin{array}{l}\text { (Janssen et } \\
\text { al., 2016) }\end{array}$ & $\begin{array}{l}\mathrm{A} 1, \mathrm{~A} 2 \mathrm{a}, \mathrm{A} 3 \\
\mathrm{~B} 1, \mathrm{~B} 2, \mathrm{~B} 3, \mathrm{Cl} \\
\mathrm{C} 2, \mathrm{D} 1, \mathrm{D} 2, \mathrm{E}\end{array}$ & 198 & $\begin{array}{l}4 \text { CR, } 21 \text { EN, } \\
46 \text { VU, } 22 \text { NT } \\
\text { y } 105 \text { LC }\end{array}$ & $\begin{array}{l}37 \text { países del } \\
\text { continente } \\
\text { europeo }\end{array}$ \\
\hline $\begin{array}{l}\text { Manglares de } \\
\text { la Isla } \\
\text { Mayotte, } \\
\text { Francia }\end{array}$ & $\begin{array}{l}\text { Regional/sistemática } \\
\text { Escala Subnacional }\end{array}$ & 3 humedales-manglares & $\begin{array}{l}\text { (lucn-France, } \\
\text { 2017) }\end{array}$ & $\begin{array}{l}\mathrm{A} 1, \mathrm{~A} 2 \mathrm{~b}, \mathrm{~A} 3, \\
\mathrm{~B} 1, \mathrm{~B} 2, \mathrm{~B} 3, \mathrm{C1}, \\
\mathrm{C} 2 \mathrm{a}, \mathrm{C} 2 \mathrm{~b}, \mathrm{C} 3, \\
\mathrm{D} 2 \mathrm{a}, \mathrm{D} 3\end{array}$ & 3 & $\begin{array}{l}1 \mathrm{EN}, 1 \mathrm{VU} \text { y } 1 \\
\mathrm{LC}\end{array}$ & Francia \\
\hline $\begin{array}{l}\text { Hotspot del } \\
\text { centro de } \\
\text { Chile }\end{array}$ & $\begin{array}{l}\text { Regional/sistemática } \\
\text { Escala Subnacional }\end{array}$ & 13 bosques y 4 matorrales & $\begin{array}{l}\text { (Alaniz et al., } \\
\text { 2016) }\end{array}$ & $\begin{array}{l}\mathrm{Al}, \mathrm{A} 3, \mathrm{~B} 1 \\
\mathrm{~B} 2, \mathrm{~B} 3\end{array}$ & 17 & $\begin{array}{l}3 \text { CR, } 3 \text { EN, } 5 \\
\text { VU, } 1 \text { NT, } 5 \text { LC. }\end{array}$ & Chile \\
\hline $\begin{array}{l}\text { Bosque cenizo } \\
\text { de montaña, } \\
\text { Australia. }\end{array}$ & Regional/estratégica & Bosque & $\begin{array}{l}\text { (Burns et al., } \\
\text { 2015) }\end{array}$ & $\begin{array}{l}\mathrm{A} 1, \mathrm{~A} 2 \mathrm{a}, \mathrm{A3} \\
\mathrm{B1}, \mathrm{B2}, \mathrm{B3}, \mathrm{D} 1, \\
\mathrm{D} 2 \mathrm{a}, \mathrm{D} 3, \mathrm{E} .\end{array}$ & 1 & CR & Australia \\
\hline
\end{tabular}

$\begin{array}{lllll}\text { (Chytrý et al., } & \text { A1, A2a, A3, } & & \text { 1 CO, 12 CR, 31 } & \\ \text { B1, B2, C1, C2, 142 } & \text { EN, 25 VU, 29 } & \text { Republica } \\ \text { 2019) } & \text { C3, D1, D2, } & \text { NTy y 44 LC } & \end{array}$




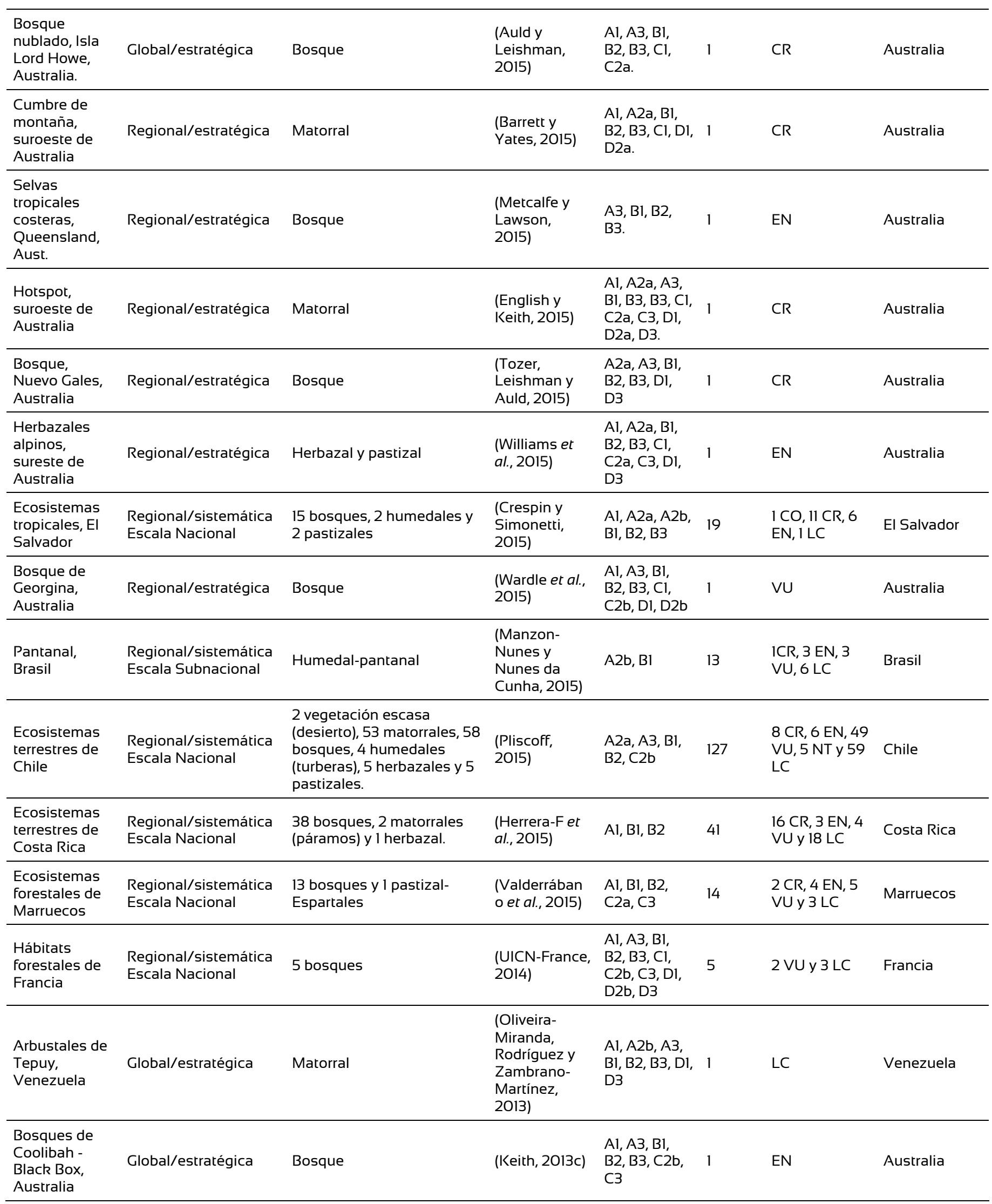




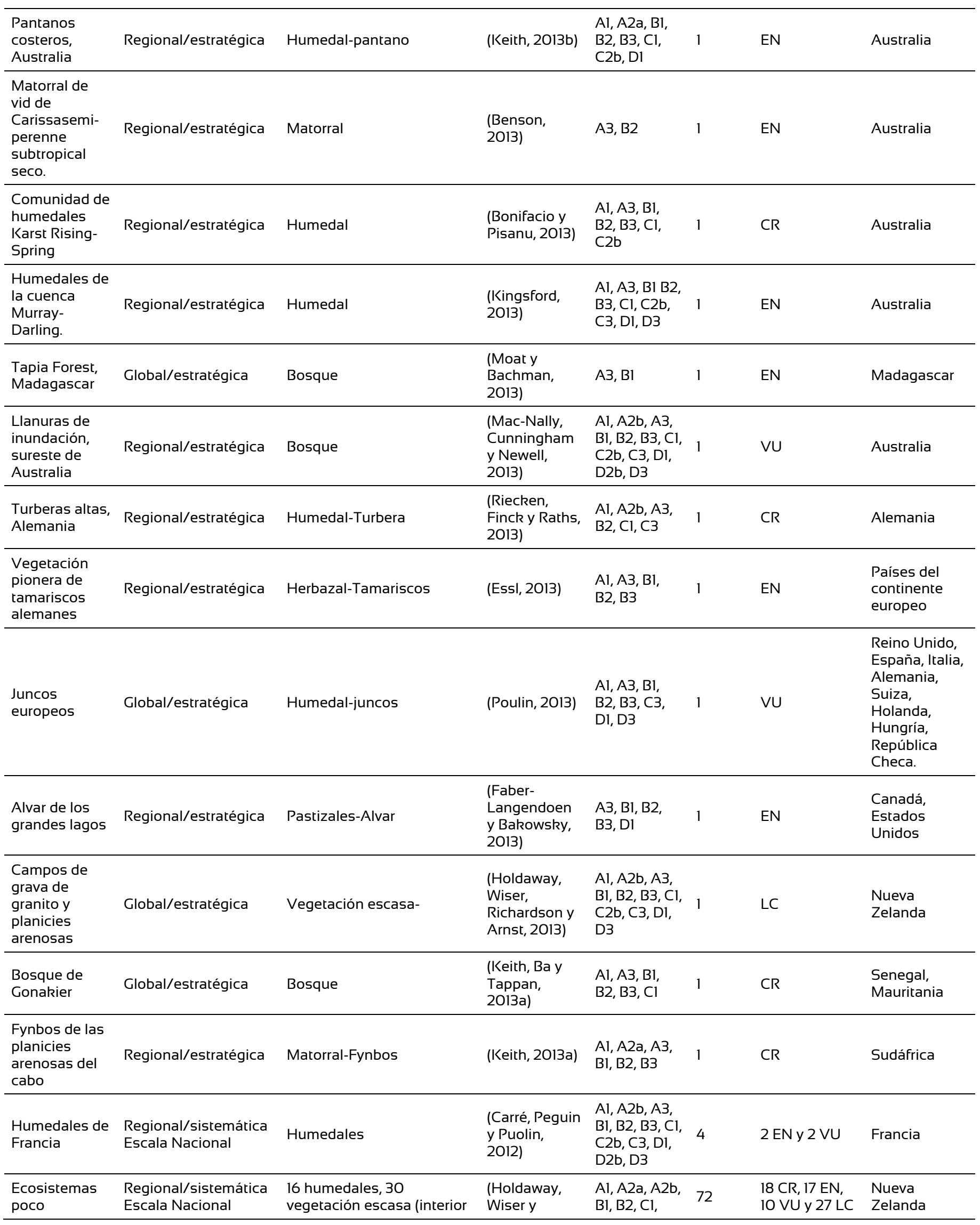




\begin{tabular}{|c|c|c|c|c|c|c|c|}
\hline $\begin{array}{l}\text { comunes, } \\
\text { Nueva } \\
\text { Zelanda }\end{array}$ & & $\begin{array}{l}\text { alpino), } 13 \text { costeros, } 13 \\
\text { ecosistemas no comunes } \\
\text { (geotérmicos y } \\
\text { subterraneos) }\end{array}$ & $\begin{array}{l}\text { Williams, } \\
\text { 2012) }\end{array}$ & $\begin{array}{l}\text { C2a, C2b, } \\
\text { D2b }\end{array}$ & & & \\
\hline $\begin{array}{l}\text { Ecosistemas } \\
\text { terrestres de } \\
\text { Venezuela }\end{array}$ & $\begin{array}{l}\text { Regional/sistemática } \\
\text { Escala Nacional y } \\
\text { subnacional }\end{array}$ & $\begin{array}{l}7 \text { bosques, } 1 \text { humedal- } \\
\text { manglar, } 5 \text { matorrales- } \\
\text { arbustales, } 5 \text { pastizales y } \\
\text { herbazales y } 1 \text { vegetación } \\
\text { escasa-saxícola }\end{array}$ & $\begin{array}{l}\text { (Oliveira- } \\
\text { Miranda et al., } \\
\text { 2010) }\end{array}$ & $\begin{array}{l}\text { A2a, C2b, } \\
D 2 b, B 2, C 2\end{array}$ & 19 & $\begin{array}{l}2 \text { CR, } 2 \text { EN, } 7 \\
\text { VU, } 1 \text { NT y } 7 \\
\text { LC. }\end{array}$ & Venezuela \\
\hline
\end{tabular}

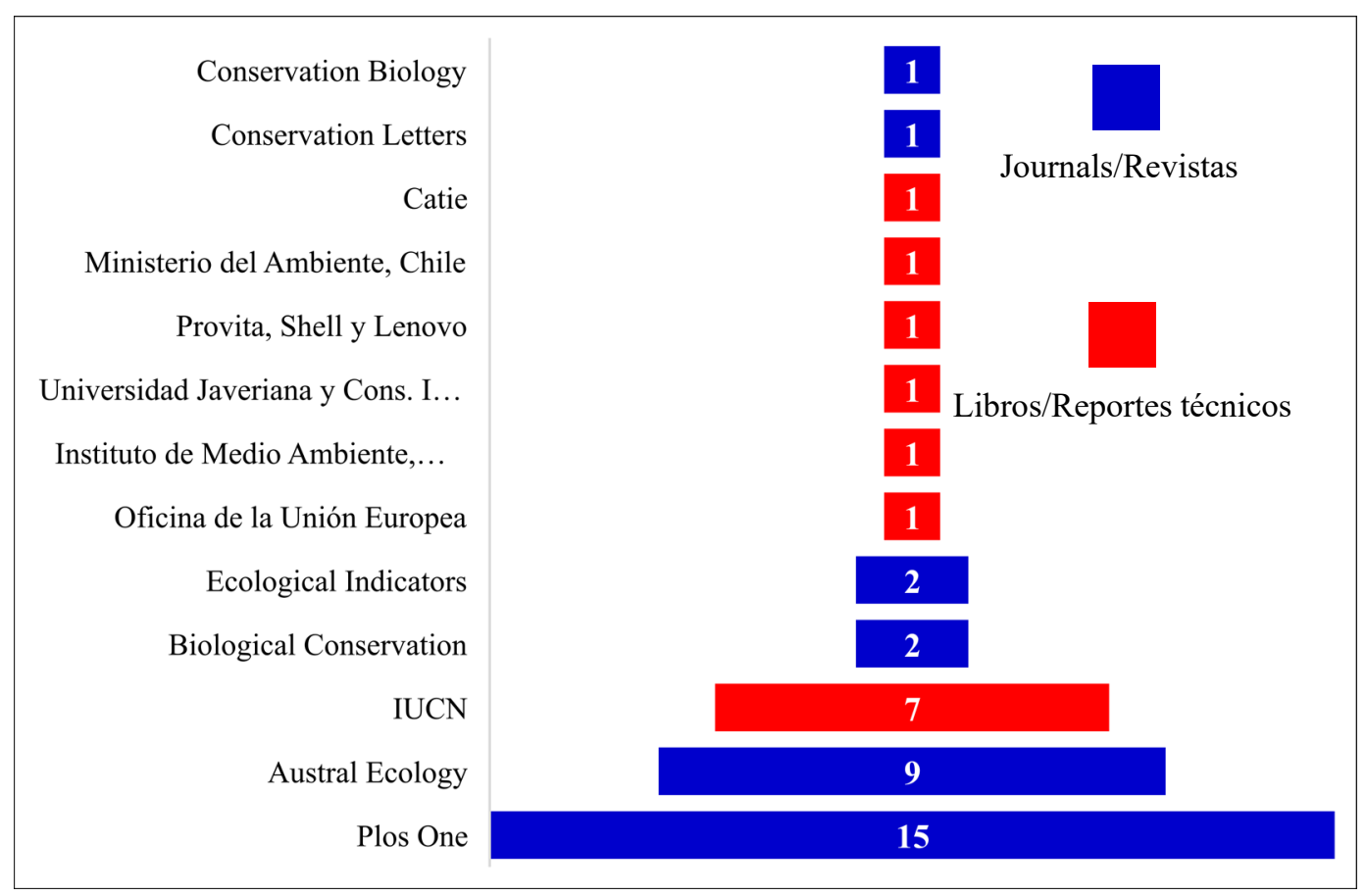

FIGURA 1. Proporción de Journals/revistas, libros y reportes técnicos relacionados con la evaluación de riego de colapso de ecosistemas terrestres y humedales.

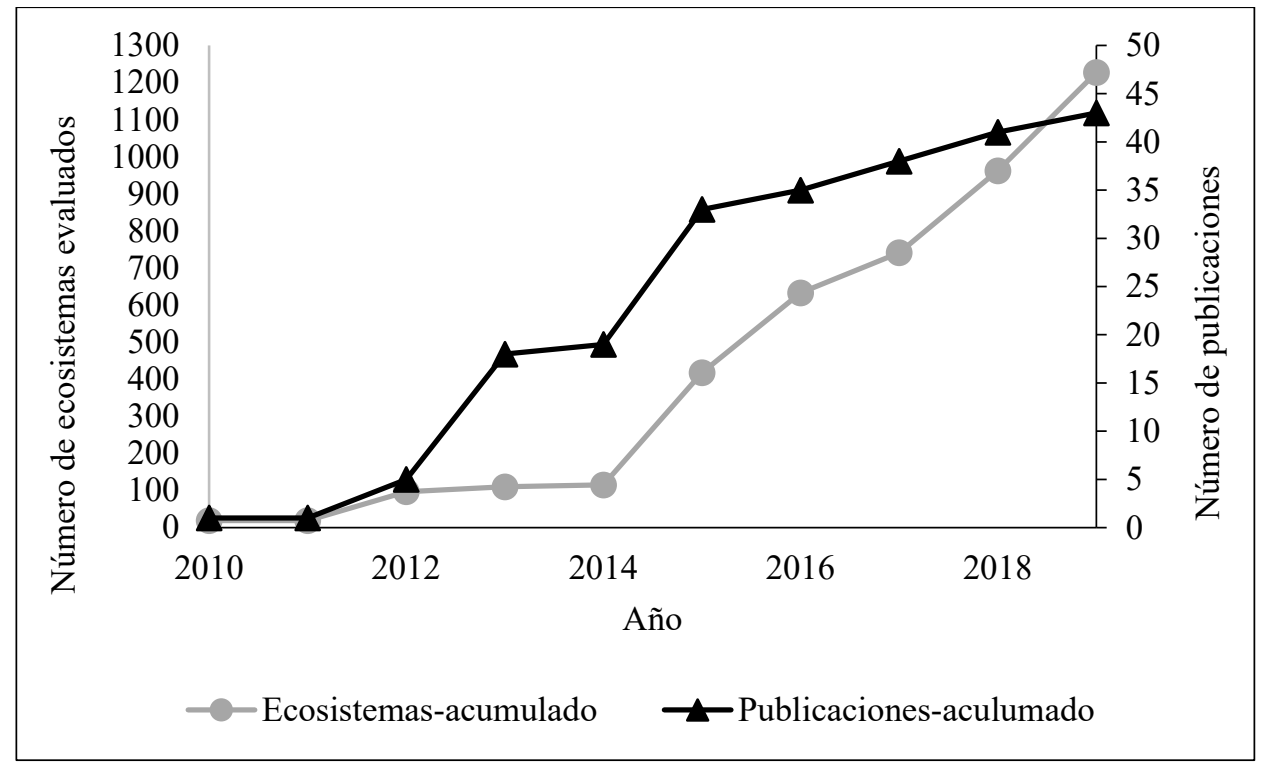

FIGURA 2. Número acumulado de publicaciones y ecosistemas evaluados por año, 2010 a 2019. 
TABLA 2. Frecuencia de las variables empleadas para la delimitación de ecosistemas.

\begin{tabular}{|c|c|c|c|c|c|c|c|c|}
\hline \multirow[b]{2}{*}{$\begin{array}{l}\text { Delimita- } \\
\text { ción del } \\
\text { ecosistema }\end{array}$} & \multirow[b]{2}{*}{$\begin{array}{l}\text { Criterio de } \\
\text { delimitación }\end{array}$} & \multicolumn{7}{|c|}{ Frecuencia de delimitación en tipos de ecosistemas } \\
\hline & & Bosques & Matorrales & $\begin{array}{c}\text { Pastizales y } \\
\text { herbazales }\end{array}$ & Humedales & Costeros & $\begin{array}{c}\text { Vegetación } \\
\text { escasa y } \\
\text { ecosistemas poco } \\
\text { comunes }\end{array}$ & Total \\
\hline Biota & $\begin{array}{l}\text { Tipo de } \\
\text { vegetación }\end{array}$ & 23 & 14 & 13 & 16 & 2 & 4 & 72 \\
\hline \multirow{10}{*}{$\begin{array}{l}\text { Factores } \\
\text { ambien- } \\
\text { tales }\end{array}$} & Temperatura & 8 & 6 & 7 & 4 & 1 & 4 & 30 \\
\hline & Precipitación & 7 & 4 & 5 & 3 & 1 & 3 & 23 \\
\hline & Altitud & 5 & 4 & 4 & - & - & - & 13 \\
\hline & Relieve & 3 & 2 & 2 & 1 & - & 2 & 10 \\
\hline & $\begin{array}{l}\text { Tipo de roca y } \\
\text { sustrato }\end{array}$ & - & - & 1 & 2 & 2 & 3 & 8 \\
\hline & $\begin{array}{l}\text { Provincia } \\
\text { fitogeográfica }\end{array}$ & 1 & 1 & 1 & - & - & - & 3 \\
\hline & Pendiente & 1 & 1 & 1 & - & - & - & 3 \\
\hline & Humedad & 1 & 1 & 1 & - & - & - & 3 \\
\hline & Ecorregión & 1 & 1 & - & - & - & - & 2 \\
\hline & $\begin{array}{l}\text { Presencia de } \\
\text { nubes }\end{array}$ & 1 & - & - & - & - & - & 1 \\
\hline Procesos & $\begin{array}{l}\text { Regímenes de } \\
\text { inundación }\end{array}$ & 2 & - & - & 16 & - & - & 18 \\
\hline \multirow{3}{*}{$\begin{array}{l}\text { División } \\
\text { adminis- } \\
\text { trativa }\end{array}$} & Frontera de país & 7 & 5 & 5 & 7 & - & 2 & 26 \\
\hline & $\begin{array}{l}\text { División } \\
\text { subnacional } \\
\text { (provincias, } \\
\text { estados, } \\
\text { municipios, } \\
\text { departamentos) }\end{array}$ & 2 & 2 & 2 & 3 & - & 1 & 10 \\
\hline & $\begin{array}{l}\text { Delimitación de } \\
\text { cuencas }\end{array}$ & 2 & 1 & 2 & 2 & - & - & 7 \\
\hline
\end{tabular}

En orden de importancia, los criterios B (distribución restringida) y A (reducción en la distribución) de la LRE fueron de aplicación general, destacan los subcriterios B1 (extensión de la presencia dada por el área de un polígono convexo mínimo que incluya todas las ocurrencias del ecosistema), el B2 (número de celdas de $10 \mathrm{~km} \times 10 \mathrm{~km}$ ocupadas) —ambos aplicados en 40 estudios-, y el A1 que calcula la disminución de la extensión en los pasados 50 años, este se utilizó en 34 publicaciones. El “criterio E” fue el menos empleado usado en solo tres estudios (Fig. 3). En $30.23 \%$ de estudios se utilizaron solamente los Criterios A y $\mathrm{B}$, en $23.26 \%$ se emplearon tres criterios (siete con la combinación $\mathrm{ABC}$ y tres $\mathrm{ABD}$ ), en $41.86 \%$ cuatro criterios (17 ABCD y uno ABDE) y en $4.65 \%$ (2 estudios) se aplicaron los cinco Criterios. En 28 estudios los periodos de datos fiables oscilan entre los 12 y 48 años $(\bar{X}=27.7$, $\mathrm{E} . \mathrm{E}=2.16, \mathrm{~S}=11.44$ ), en estos casos se emplearon técnicas de modelación estadística para cubrir los periodos requeridos por la LRE. Hubo 412 ecosistemas clasificados como LC, 125 NT, 281 VU, 226 EN, 181 CR y dos CO. No se encontró una diferencia significativa entre los ecosistemas "amenazados" (56.07\%) y "no amenazados" $(43.77 \%)(\mathrm{W}=5650, \mathrm{P}=0.7914)$. 
TABLA 3. Frecuencia de indicadores y variables para medir colapso.

\begin{tabular}{|c|c|c|c|c|c|c|c|c|}
\hline \multirow[b]{2}{*}{$\begin{array}{l}\text { Indicadore } \\
\text { s de } \\
\text { colapso }\end{array}$} & \multirow[b]{2}{*}{ Variable empleada } & \multicolumn{7}{|c|}{ Frecuencia en la que se empleó la variable en cada tipo de ecosistemas } \\
\hline & & Bosques & Matorrales & $\begin{array}{l}\text { Pastizales } \\
\text { y } \\
\text { herbazales }\end{array}$ & Humedales & Costeros & $\begin{array}{l}\text { Veg. escasa y } \\
\text { ecosistemas } \\
\text { poco } \\
\text { comunes }\end{array}$ & Total \\
\hline Espaciales & $\begin{array}{l}\text { Cambio en la cobertura } \\
\text { vegetal, extensión y } \\
\text { distribución. }\end{array}$ & 23 & 14 & 12 & 16 & 2 & 5 & 72 \\
\hline \multirow{15}{*}{ Bióticos } & $\begin{array}{l}\text { Invasión de especies } \\
\text { exóticas (flora y fauna) }\end{array}$ & 4 & 4 & 5 & 5 & 1 & 2 & 21 \\
\hline & $\begin{array}{l}\text { Cambios en la distribución, } \\
\text { riqueza y abundancia de } \\
\text { especies clave }\end{array}$ & 5 & 3 & 3 & 4 & 1 & - & 16 \\
\hline & $\begin{array}{l}\text { Densidad de población } \\
\text { humana }\end{array}$ & 2 & - & 1 & 1 & - & - & 4 \\
\hline & $\begin{array}{l}\text { Producción y/o pérdida de } \\
\text { biomasa }\end{array}$ & 2 & 1 & 1 & - & - & - & 4 \\
\hline & $\begin{array}{l}\text { Cambio en la composición } \\
\text { de especies sucesoras }\end{array}$ & 3 & 2 & - & . & - & - & 5 \\
\hline & $\begin{array}{l}\text { Declinación en la } \\
\text { producción primaria }\end{array}$ & - & - & - & 2 & - & - & 2 \\
\hline & $\begin{array}{l}\text { Declinación en los taxones } \\
\text { de flora nativa }\end{array}$ & 2 & 2 & - & - & - & - & 4 \\
\hline & $\begin{array}{l}\text { Degradación del estrato } \\
\text { arbóreo/arbustivo }\end{array}$ & 2 & 1 & - & - & - & - & 3 \\
\hline & $\begin{array}{l}\text { Infestación por } \\
\text { fitopatógenos }\end{array}$ & 1 & 1 & - & - & - & - & 2 \\
\hline & $\begin{array}{l}\text { Aumento en la presión de } \\
\text { herbívoros }\end{array}$ & 1 & - & - & - & - & - & 1 \\
\hline & $\begin{array}{l}\text { Defaunación de grandes } \\
\text { mamíferos }\end{array}$ & 1 & - & - & - & - & - & 1 \\
\hline & $\begin{array}{l}\text { Degradación de individuos } \\
\text { pioneros }\end{array}$ & - & - & - & 1 & - & - & 1 \\
\hline & Mortandad de árboles & 1 & - & - & - & - & - & 1 \\
\hline & $\begin{array}{l}\text { Número de huecos } \\
\text { (madrigueras) en árboles }\end{array}$ & 1 & - & - & - & - & - & 1 \\
\hline & $\begin{array}{l}\text { Reducción en la dispersión } \\
\text { de semillas }\end{array}$ & 1 & - & - & - & - & - & 1 \\
\hline \multirow[b]{2}{*}{ Abióticos } & $\begin{array}{l}\text { Variables bioclimáticas: } \\
\text { escenarios cambio climático }\end{array}$ & 9 & 4 & 2 & 4 & 2 & 2 & 23 \\
\hline & $\begin{array}{l}\text { Cambio hidrológico: altura } \\
\text { de diaria de rio, cambio en } \\
\text { nivel freático, régimen de }\end{array}$ & 3 & 1 & - & 9 & - & - & 13 \\
\hline
\end{tabular}


flujo y volúmenes de descarga.

\begin{tabular}{|c|c|c|c|c|c|c|c|}
\hline $\begin{array}{l}\text { Explotación forestal, } \\
\text { sobrepastoreo o cambio en } \\
\text { el uso de suelo }\end{array}$ & 9 & 2 & 3 & - & - & - & 14 \\
\hline $\begin{array}{l}\text { Disponibilidad de agua: } \\
\text { precipitación y } \\
\text { evapotranspiración }\end{array}$ & 3 & 2 & 1 & - & - & - & 6 \\
\hline $\begin{array}{l}\text { Grado de intervención } \\
\text { antropogénica/ extracción } \\
\text { de recursos }\end{array}$ & 3 & 2 & 2 & 2 & - & 2 & 11 \\
\hline $\begin{array}{l}\text { Índice de estrés hídrico y } \\
\text { térmico }\end{array}$ & 2 & 1 & 1 & 1 & - & & 5 \\
\hline $\begin{array}{l}\text { Métricas de paisaje: } \\
\text { fragmentación, pérdida de } \\
\text { conectividad. }\end{array}$ & 2 & 1 & 1 & - & - & & 4 \\
\hline $\begin{array}{l}\text { Erosión, degradación, } \\
\text { pisoteo o perturbación } \\
\text { mecánica de suelo }\end{array}$ & 2 & - & 2 & - & - & 2 & 6 \\
\hline Intervalos de incendios & 2 & 1 & - & - & - & & 3 \\
\hline $\begin{array}{l}\text { Fertilidad y capacidad de } \\
\text { suelos para agricultura }\end{array}$ & 1 & - & 1 & - & - & & 2 \\
\hline $\begin{array}{l}\text { Profundidad de la capa de } \\
\text { nieve (snowpack) }\end{array}$ & - & - & 2 & - & - & & 2 \\
\hline $\begin{array}{l}\text { Declinación en las prácticas } \\
\text { tradicionales de manejo }\end{array}$ & 1 & - & - & - & - & - & 1 \\
\hline $\begin{array}{l}\text { Acidificación por deposición } \\
\text { atmosférica/ Concentración } \\
\text { de ozono troposférico }\end{array}$ & 3 & - & - & - & - & - & 3 \\
\hline $\begin{array}{l}\text { Deportes al aire libre y } \\
\text { actividades recreativas } \\
\text { (incluyendo alpinismo y } \\
\text { esquí) }\end{array}$ & - & - & - & - & - & 1 & 1 \\
\hline $\begin{array}{l}\text { Disminución en la } \\
\text { frecuencia en la cobertura } \\
\text { de nubes }\end{array}$ & 1 & - & - & - & - & - & 1 \\
\hline $\begin{array}{l}\text { Erosión de los frentes del } \\
\text { manglar }\end{array}$ & - & - & - & 1 & - & - & 1 \\
\hline $\begin{array}{l}\text { Eutroficación, concentración } \\
\text { de nutrientes y sustancias } \\
\text { contaminantes }\end{array}$ & 1 & 2 & 2 & 8 & 2 & 4 & 19 \\
\hline $\begin{array}{l}\text { Extensión de las zonas } \\
\text { inundadas }\end{array}$ & - & - & - & 1 & - & - & 1 \\
\hline
\end{tabular}




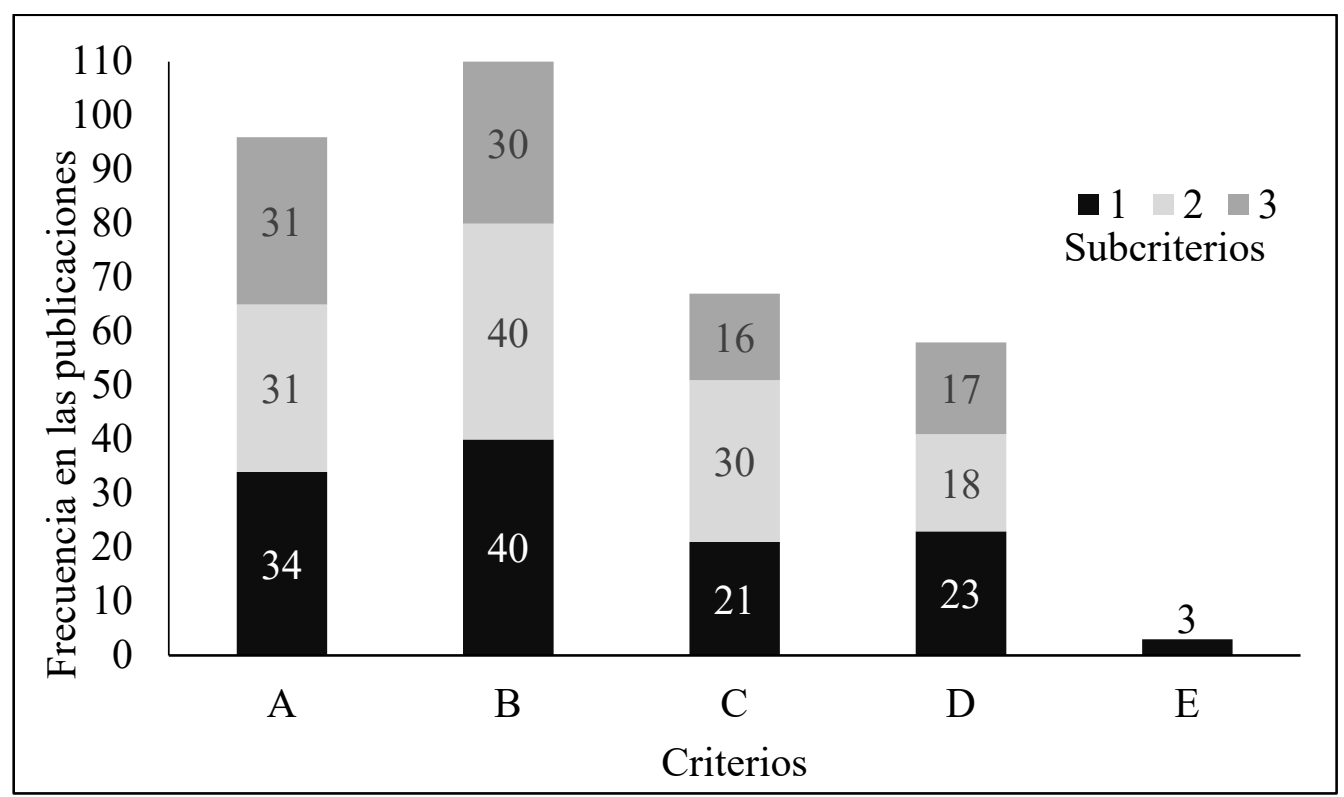

FIgURA 3. Criterios y subcriterios de la Lista Roja de Ecosistemas de la Unión Internacional para la Conservación de la Naturaleza empleados en las evaluaciones de riesgo.

Las evaluaciones de riesgo incluyeron 69 países. El mayor número de publicaciones lo tuvo Australia con 14 y la República Checa con el mayor número de ecosistemas evaluados en su territorio con 341 (Fig. 4). En 10 países se han llevado a cabo evaluaciones estratégicas de un ecosistema por vez, en 22 países se han realizado evaluaciones sistemáticas de uno a 17 ecosistemas simultáneamente, en 13 países se han implementado evaluaciones estratégicas nacionales donde se abarcó el total del territorio y 57 países forman parte de dos evaluaciones continentales: 23 de bosques de América y 24 de ecosistemas terrestres de Europa (Tabla 1).

\section{DISCUSIÓN}

\section{Tendencias en las publicaciones}

Del total de estudios, $72.09 \%$ están en 3/13 editoriales, la principal fue Plos One (34.88\%) donde se publicó el artículo de Keith et al. (2013b) que además de sustentar teóricamente la LRE, compiló 20 evaluaciones, de ellas diez fueron en ecosistemas terrestres y cinco en humedales. No obstante, Austral Ecology (20.93\%) -segunda en importanciatuvo más artículos en ediciones independientes (Fig. 1). Probablemente, la tendencia de concentrar información en pocas editoriales se deba a que los autores se interrelacionan entre sí en pocos grupos de trabajo, formando una red con alta modularidad. Esta hipótesis requiere un análisis de redes que identifique individuos y equipos que jueguen roles centrales en la generación de conocimiento, así como elementos aislados que necesitan más apoyo en las evaluaciones de riesgo. Con ello se pueden generar estrategias para acelerar flujos de conocimiento, canales de comunicación y optimización de recursos (Serrat, 2017).

La mayoría de las editoriales (53.8\%) no están dentro del JCR, aunque solo reúnen $33.23 \%$ de las publicaciones y corresponden a evaluaciones nacionales y una continental (Tabla 1). Se publicaron en libros o reportes editados por instituciones de gobierno, universidades y, sobre todo, la IUCN quien fue la tercera fuente en importancia (16.28\%) (Fig. 1). En ellos no se siguió un procedimiento formal de arbitraje, fueron documentos de aplicación técnica que respondieron a necesidades prácticas de conservación. Quizá los próximos trabajos se deban orientar a nuevos experimentos, modelos y métodos que refuercen el sustento teórico de la LRE, y se refleje en un incremento en las evaluaciones ya que en los últimos cuatro años solo se han realizado 10/43 publicaciones y solamente cuatro corresponden a artículos del JCR (Fig. 2). 


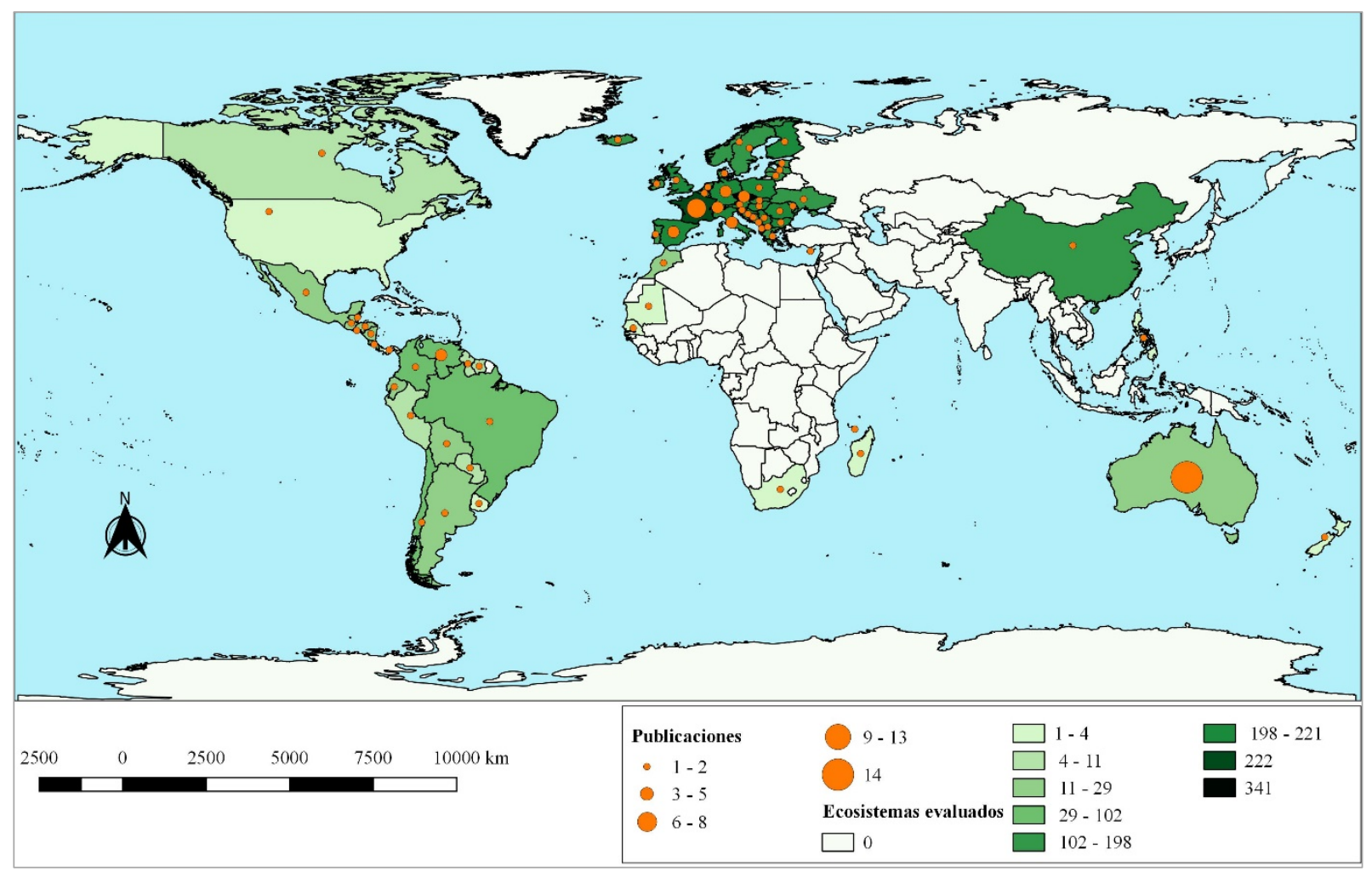

FIGURA 4. Número de publicaciones y ecosistemas evaluados por país bajo los protocolos de la Lista Roja de Ecosistemas de la Unión Internacional para la Conservación de la Naturaleza, 2010 a 2019.

Del total de ecosistemas, $73.43 \%$ se agruparon en $6 / 43$ publicaciones realizadas del 2015 al 2019, en contraste 24/43 estudios indicaron la evaluación de uno solo (Fig. 2). Se identificaron dos periodos de acuerdo con número y tipo de evaluaciones, el primero de 2010 a 2014 donde cada una analizó en promedio 6.05 ecosistemas; fueron principalmente "evaluaciones estratégicas" de diagnóstico detallado, dirigidas a uno o pocos tipos de ecosistemas y orientadas a desarrollar estrategias de gestión (Bland et al., 2019). Además, se desarrollaron 6/7 evaluaciones "globales" aplicadas a la extensión total de ecosistemas de distribución única, es decir, endémicos cuya pérdida significaría su desaparición total (Tabla 1). El segundo de 2015 a 2019, el promedio aumentó a 42.17 ecosistemas por estudio, se analizó $90.63 \%$ de ecosistemas con una tendencia anual de crecimiento (Fig. 2). Principalmente se desarrollaron evaluaciones "sistemáticas" dirigidas a todos los ecosistemas dentro de un área para informar sus riesgos relativos (Bland et al., 2019). A escala continental se realizaron para identificar áreas prioritarias para la asignación de recursos internacionales (Ferrer-Paris et al., 2019), reconocer presiones, amenazas, estados de conservación, restauración de partes del ecosistema, y monitorear del cumplimiento de objetivos internacionales sobre compromisos de biodiversidad (Janssen et al., 2016). A nivel nacional y subnacional contribuyeron con los gobiernos para implementar políticas de acción y conservación, abordarlos vacíos de representatividad de ecosistemas que no se encuentran dentro áreas protegidas y establecer áreas prioritarias de conservación o restauración (Tabla 1) (Rodríguez et al., 2010; Herrera-F et al., 2015).

\section{Tipos de ecosistemas, extensión y criterios de delimitación}

Los ecosistemas abarcaron $43.50 \%$ de los referidos por Bland et al. (2019) a nivel mundial, quienes además 
incluyeron dulceacuícolas, marinos y registrados en documentos no publicados (evaluaciones en progreso o suspendidas). Los bosques fueron los ecosistemas más evaluados, representan $50.01 \%$ de los terrestres y $43.28 \%$ del total (incluyendo humedales), se analizaron en 11 de las 20 evaluaciones sistemáticas y en nueve de las 23 estratégicas (Tabla 1). En contraste los humedales abarcan $13.53 \%$, sin embargo, estuvieron en 12 evaluaciones sistemáticas y cinco estratégicas.

Solamente $22.33 \%$ de los ecosistemas indicaron su extensión, el promedio $\left(9015.85 \mathrm{~km}^{2}\right)$ no representó una tendencia en su tamaño, ya que $50 \%$ de ellos son menores a $712.40 \mathrm{~km}^{2}$, además, existió una gran variabilidad en el área $\left(S=31\right.$ 204.16), el intervalo fue de $0.28 \mathrm{~km}^{2}$ del "Bosque Nublado de la Isla Lord Howe" en Australia (Auld y Leishman, 2015) a $311496.00 \mathrm{~km}^{2}$ del "Bosque Siempreverde" de Venezuela (Oliveira-Miranda et al., 2010). En trabajos donde se evaluaron a diferentes escalas el tamaño influyó en las categorías de riesgo, por ejemplo, en Finlandia, Marruecos y Venezuela existieron ecosistemas con una categoría a escala de país y con categorías contrastantes en partes de sus subdivisiones (OliveiraMiranda et al., 2010; Valderrábano et al., 2015; Kontula y Raunio, 2018). Las condiciones de escala influyen directamente en las categorías de riesgo, ya que los procesos que ocurren a una escala de paisaje no pueden verse localmente, y el deterioro local puede enmascararse en una escala mayor (O'Neill, 2001).

Sato y Lindenmayer (2018) identificaron dos temas prioritarios a resolver en el análisis de riesgo: la delimitación del ecosistema y la conceptualización de su colapso. La LRE sugiere su definición basándose en cuatro elementos propuestos por Tansley (1935): un complejo biótico, un ambiente abiótico, las interacciones dentro y entre ellos, y un espacio físico (Pickett y Cadenasso, 2002; Bland et al., 2016), dejando a criterio de los investigadores el uso de variables de delimitación, tamaños y escalas. Se encontró que el tipo vegetación fue el elemento en común para definirlos, en el caso de ecosistemas terrestres frecuentemente se combinó con intervalos de temperatura, precipitación y altitud, y en humedales con los regímenes de inundación. Además, la determinación con base en límites fronterizos o unidades administrativas al interior de los países fueron factores que no está en función de las dinámicas bióticas, abióticas o de los procesos del ecosistema, pero su uso fue frecuente para acotarlos (Tabla 2).

La vegetación como proxy del ecosistema se abordó desde diferentes enfoques. A nivel continental, Europa utilizó hábitats tipo de la EUNIS (European Nature Information System) basados en características fitosociológicas (Janssen et al., 2016), mientras que en América se empleó un enfoque botánico-ecológico de los bosques templados y tropicales (Ferrer-Paris et al., 2019). A escala nacional se aplicaron clasificaciones oficiales, por ejemplo, el mapa de ecosistemas de China se basó en especies dominantes (Tan et al., 2017), Chile los definió como comunidades vegetales con estructura uniforme y fisonomía en condiciones climáticas particulares (Pliscoff, 2015; Alaniz, Galleguillos y Perez-Quezada, 2016), Costa Rica empleó unidades fitogeográficas (Herrera-F et al., 2015), Venezuela uso aspectos botánicos y ecológicos interactuado con variables ambientales (Oliveira-Miranda et al., 2010; Rodríguez et al., 2010) y en República Checa se empleó la fisonomía y formaciones dominantes (Chytrý et al., 2019).

\section{Variables de colapso, criterios de la LRE utilizados y marcos temporales}

Las métricas del cambio en la cobertura vegetal, extensión y distribución del ecosistema — variables directamente relacionadas con su estructura y composición (Crespin y Simonetti, 2015; Alaniz et al., 2016) — se aplicaron en todos los casos para evaluar los criterios A (reducción en distribución) y B (distribución restringida) de la LRE (Tabla 3). Probablemente porque la desaparición espacial es una condición plausible que da certeza del punto final de los ecosistemas (Keith et al., 2013b), y porque el cambio de uso de la tierra ha tenido el mayor impacto negativo sobre la naturaleza desde 1970 (Ipbes, 2019). Los resultados concuerdan con lo obtenido por Rowland et al. (2018) 
donde los indicadores espaciales fueron los más utilizados en ecosistemas terrestres. No obstante, ellos encontraron que sobresale la aplicación el "Criterio A" a diferencia de este estudio donde se encontró mayor aplicación del "Criterio B" (Fig. 3).

Las variables relacionadas a escenarios de cambio climático fueron las más frecuentes encontradas para aplicar el "criterio C" de la LRE (degradación biótica). Otras variables bióticas destacadas fueron el cambio de uso de suelo, explotación forestal o sobrepastoreo aplicados en ecosistemas terrestres y en el cambio hidrológico en los humedales. Respecto a las variables bióticas empleadas en el "criterio D", las más utilizadas fueron la invasión de especies exóticas de flora y fauna, y el cambio en la distribución, riqueza y abundancia de especies clave (Tabla 3). Al igual que en el estudio de Rowland et al. (2018), después de los indicadores espaciales (Criterio A y B) el Criterio C se aplicó con mayor frecuencia (Fig. 3).

En algunos estudios se emplearon variables específicas para determinar estados de colapso, por ejemplo: Burns et al. (2015) determinaron que el intervalo de incendios naturales influyeron en las dinámicas de un Bosque Cenizo en Australia, el grado de afectación de patógenos en especies forestales en un ecosistema de cumbre de Montaña en Australia (Barrett y Yates, 2015), la caída del número de días nublados por año y la precipitación anual en el Bosque Nubado de GnarledMossy en Australia (Auld y Leishman, 2015), la perturbación mecánica del suelo del Alvar en los Grandes Lagos de Norteamérica (Faber-Langendoen y Bakowsky, 2013) y la presión del turismo en ecosistemas de la República Checa (Chytrý et al., 2019). Algunas variables que fueron particulares en humedales contemplan la productividad primaria en manglares (Marshall, Schulte to Bühne, Bland y Pettorelli, 2018) y cambios en los flujos hidrológicos e inundaciones (Bonifacio y Pisanu, 2013; Keith, 2013c; Kingsford, 2013) (Tabla 3).

Solo en dos trabajos se emplearon los cinco Criterios de la LRE y en más de la mitad (53.49\%) se utilizaron hasta tres (Tabla 1). Evaluar los ecosistemas con el mayor número criterios daría más certeza del riesgo de colapso (Sato y Lindenmayer, 2018), no obstante, la LRE considera suficiente la evaluación de alguno para asignar una categoría de riesgo (Bland et al., 2017a). Al igual que lo señalado por Rowland et al. (2018), el "criterio E” fue el menos frecuente, se aplicó únicamente en tres estudios a pesar de ser el más determinante para estimar la probabilidad de colapso mediante modelos cuantitativos (Sato y Lindenmayer, 2018) (Fig. 3). Su limitada aplicación probablemente se debe a la falta de datos para generar predicciones confiables y robustas, dejando un campo de estudio para generar modelos experimentales que den mayor certeza a los estados de riesgo (Boitani, Mace y Rondinini, 2015; Sato y Lindenmayer, 2018). En contraste, Los subcriterios B1 y B2 fueron los más empleados debido a que no se emplean modelos o series temporales para su medición, evalúa la condición actual del ecosistema siempre y cuando haya un proceso de amenaza explícita.

Del total de estudios, $65.17 \%$ abarcaron de 12 a 48 años de datos confiables, lapso suficiente para detectar alertas tempranas de colapso que se manifiestan en un intervalo de 1 a 40 años (Sato y Lindenmayer, 2018). Sin embargo, para cumplir con el marco temporal establecido en la LRE se usaron tasas de disminución absoluta (ARD) y tasas de disminución proporcional (PRD) (Tan et al., 2017; Marshall et al., 2018), estimadas a partir de series temporales cuyos datos se extrapolaron con modelos de funciones exponenciales (PRD) o lineales (ARD) (Bland et al., 2017a). También usaron modelos de reconstrucción del ecosistema (Ferrer-Paris et al., 2019), así como un porcentaje de calidad en la evaluación temporal (p. ej. si se trabajaba con 40 años de datos, la calidad de la evaluación de declaró en el 80\%) (Alaniz et al., 2016). Las imágenes de satélite fueron de los principales insumos; sin embargo, su aparición data de 1972, por lo que a futuro se tendrán más series de datos confiables que cumplan con el periodo establecido en la LRE (Alaniz et al., 2016).

\section{Estados de Riesgo}

Tres cuartas partes de la superficie terrestre tiene algún grado de alteración, los ecosistemas se han reducido en promedio $47 \%$ de su estado natural y muchos de ellos 
siguen disminuyendo más de 4\% por década, destacan los humedales que perdieron más de $85 \%$ de extensión (Ipbes, 2019). A pesar de ello, no se encontró diferencias significativas entre ecosistemas amenazados y noamenazados, además, la categoría LC tuvo mayor frecuencia y solo se encontraron dos colapsados. No obstante, la cantidad de publicaciones y ecosistemas analizados son muy pocos para tener una tendencia global, los estudios representan apenas $0.29 \%$ comparados con los 15000 trabajos analizados por la Ipbes (2019) para establecer tendencias globales sobre la biodiversidad y servicios ecosistémicos. Conforme se incrementen las evaluaciones de riesgo en los próximos años, se tendrá mayor certeza del estado global de los ecosistemas terrestres y humedales.

Otro motivo probable de encontrar categorías de riesgo bajo es porque los modelos construidos con periodos de datos confiables menores a 50 años pudieron subestimar la situación real de los ecosistemas, ya que a partir de 1990 algunos como los bosques tropicales y subtropicales han aumentado su extensión en ciertos países, y los bosques templados y boreales se han incrementado a nivel mundial (Ipbes, 2019), este incremento reciente pudo modificar la tendencia total.

Asimismo, el colapso ecosistémico se puede dar a través de múltiples formas y exhibir diferentes síntomas de degradación (Bland et al., 2018); una categoría derivada de los Criterios A y B (variables espaciales) con los que se evaluaron todos los ecosistemas-y $33.41 \%$ se analizó exclusivamente con ellos- probablemente subestimó el riesgo dado por otros conductores de tipo biótico o abiótico. A pesar del incremento en extensión de algunos ecosistemas, desde 1990 la abundancia de especies de biomas terrestres disminuyó al menos 20\% (Ipbes, 2019). Aproximadamente $25 \%$ de especies de plantas y animales están amenazados, un millón de especies enfrentan la extinción, muchas de ellas impactadas por especies exóticas invasoras; especialmente los vertebrados han disminuido considerablemente en los últimos 50 años, condiciones que afectan los procesos ecosistémicos (Ipbes, 2019). Por ejemplo, Redford (1992) determinó que ecosistemas con excelente conservación de vegetación no garantizó la presencia de fauna residente que se ve afectada por procesos de extracción selectiva. Es necesario que se incrementen el número de evaluaciones analizadas con los Criterios C, D y, especialmente, el “criterio E”, para dar una condición más plausible del estado de riesgo ecosistémico.

\section{Distribución geográfica de publicaciones y ecosistemas evaluados}

Los países involucrados representan 35.57\% de los países del planeta y $69 \%$ de los 100 referidos por Bland et al. (2019), quienes además de ecosistemas terrestres y humedales, incluyeron ecosistemas marinos y ecosistemas evaluados en documentos aún no publicados. Destacaron las evaluaciones continentales de la Lista Roja de Hábitats de Europa (Janssen et al., 2016) y la de Bosques Templados y Tropicales de América (Ferrer-Paris et al., 2019) que en conjunto abarcaron 57 países. Los continentes menos representados fueron África y Asia, es necesario trazar las líneas de trabajo para aplicar evaluaciones en esta zona para contribuir a la meta que se propuso la IUCN en la que estableció que todos los ecosistemas del mundo estarían evaluados para el 2025 (Keith et al., 2013b; Bland et al., 2017a). El análisis espacial aquí realizado da una idea de los vacíos de representatividad de las evaluaciones globalmente (Fig. 4).

A pesar de que Australia no alberga el mayor número de ecosistemas evaluados, en su territorio se han llevado a cabo 14 evaluaciones estratégicas no solo para desarrollar la planificación de manejo de los ecosistemas, sino también para generar conocimiento acerca de los criterios y procesos de evaluación de colapso. Esto se ha reflejado en los documentos clave como "Los Fundamentos Científicos de la LRE" (Keith et al., 2013b) y "Las Directrices para la Aplicación de la LRE” (Bland et al., 2017a). En contraste, La República Checa ostenta el mayor número de ecosistemas evaluados en tres estudios: uno continental, uno nacional y uno sistemático (Fig. 4). 


\section{CONCLUSIONES}

La LRE de la IUCN fue desarrollada por investigadores para evaluar el riesgo de colapso de los ecosistemas, apoya a los gobiernos y tomadores de decisiones en la gestión de la biodiversidad, la implementación de políticas de conservación y la protección de ecosistemas. No obstante, en los últimos cuatro años disminuyeron sus publicaciones que evalúan ecosistemas terrestres y humedales, especialmente las difundidas en revistas JCR. Se sugiere incrementar el número trabajos orientados a la generación de nuevos métodos, experimentos y modelos que refuercen las bases teóricas de la LRE y proporcionen nuevos insumos para futuras evaluaciones. Asimismo, es necesario analizar la red de colaboradores de la LRE para detectar equipos de trabajo, elementos clave, nodos aislados, flujos de difusión de conocimiento y con base en ello se puede trazar estrategias y rutas para potenciar la aplicación de la LRE.

Las evaluaciones se han realizado a niveles continentales, nacionales o subnacionales (estados, provincias, departamentos), de acuerdo con los objetivos de conservación de cada nivel, aunque muy pocos estudios indican un análisis multiescalar en un mismo territorio. No se ha documentado el empleo de la LRE en sitios donde los ecosistemas tienen una gran variabilidad en áreas pequeñas con mosaicos intrincados ambientalmente y que están ligados a una compleja diversidad sociocultural (Bray, Duran y Molina, 2012). Aquí el análisis debe incluir escalas sumamente detalladas donde la delimitación de los ecosistemas considere las necesidades locales de conservación, las dinámicas en la toma de decisiones sobre los recursos naturales y las prácticas comunitarias de reconocimiento del territorio.

La poca comparabilidad de las unidades de evaluación de la LRE ha sido cuestionada por algunos autores (Boitani et al., 2015). En este análisis se encontraron 15 criterios para definirlos, aunque el elemento común fue la vegetación usada como proxy de su ocurrencia. Aun así, incluso usando este solo elemento, fue abordado desde diferentes enfoques (botánico, ecológico, fitosociológico y fitogeográfico), el resultado fueron ecosistemas con una gran variabilidad en su definición, tamaños y escalas de análisis. Actualmente la LRE trabaja en una tipología de ecosistemas (Bland et al., 2017a), es necesario que en ella se considere una definición estandarizada aplicable a diferentes escalas y que permita realizar evaluaciones anidadas en diferentes jerarquías del ecosistema. El análisis a una sola escala no satisface las necesidades de quienes tomas las decisiones en la gestión del ecosistema a distintos niveles (Hassan et al., 2005; Sato y Lindenmayer, 2018).

Los procesos hacia el colapso pueden darse por diferentes vías y en diferentes lapsos temporales (Rowland et al., 2018; Sato y Lindenmayer, 2018). Sin embargo, todos los estudios se enfocaron a la medición de variables espaciales: pérdida de cobertura, disminución de extensión y restricciones en la distribución (Criterios A y B), en menor grado se midieron variables bióticas (Criterio D) y abióticas (Criterio C), y solo 3/43 estudios evaluaron el Criterio E que da más certeza matemático-probabilística del colapso. Es necesario que los investigadores consideren en futuras evaluaciones la inclusión de un mayor número de criterios que permita medir de forma más integral el nivel relativo de amenaza de los ecosistemas, incluso se sugiere la evaluación rigurosa bajo todos los criterios partiendo de un punto de referencia del colapso (Boitani et al., 2015; Rodríguez et al., 2015; Sato y Lindenmayer, 2018).

En la mayoría de los estudios se encontraron restricciones en la disponibilidad de datos fiables que cubrieran los periodos establecidos por la LRE, en esos casos se usaron modelos de estimación con cierto margen de incertidumbre basados en series temporales y sus tendencias. En el futuro, se tendrá más certeza de los estados de riesgo al contar con un mayor periodo de datos fiables, no obstante, para ello es fundamental realizar el monitoreo constante de las variables que determinaron los estados de riesgo actuales y combinarlas con nuevas variables en modelos que arrojen una categoría de amenaza más precisa.

Los estudios realizados no representan una tendencia global del estado de los ecosistemas terrestres y humedales debido a que son muy pocos para establecer un juicio de 
esta condición. Por esta razón los resultados contrastan con el panorama mundial que sugiere la modificación de 75\% de los ecosistemas (Ipbes, 2019). El análisis realizado arrojó el predominio de la categoría LC (Preocupación Menor) en los ecosistemas, además de no encontrar diferencias significativas entre ecosistemas amenazados y noamenazados, aunado a que solo se registraron dos ecosistemas colapsados. Con las consideraciones sugeridas y el incremento en el número de evaluaciones se espera que en un futuro se tenga un panorama que se ajusten a las tendencias globales de la biodiversidad y los ecosistemas.

Por último, el análisis geográfico realizado identifica el vacío territorial en los países donde no se han realizado las evaluaciones de riesgo de la LRE, puede ser una herramienta que apoye la priorización de naciones por evaluar. Asimismo, da una perspectiva muy general del grado de avance del cumplimento el cumplimiento de la meta global de evaluar los ecosistemas en 2025.

\section{RECONOCIMIENTOS}

Se reconoce el apoyo de la Secretaría de Investigación y Posgrado del IPN (SIP; 20200713). CAMG agradece al Consejo Nacional de Ciencia y Tecnología (Conacyt) por la beca 298180. MB-S agradece a la Comisión de Operación y Fomento a las Actividades Académicas (Cofaa) y al Programa de Estímulos al Desempeño a la Investigación (EDI) del IPN por su apoyo, así como al Sistema Nacional de Investigadores (SNI).

\section{REFERENCIAS}

Alaniz, A. J., Galleguillos, M., \& Perez-Quezada, J. F. (2016). Assessment of quality of input data used to classify ecosystems according to the IUCN Red List methodology: The case of the central Chile hotspot. Biological Conservation, 204, 378-385. doi: 10.1016/j.biocon.2016.10.038

Auld, T. D., \& Leishman, M. R. (2015). Ecosystem risk assessment for Gnarled Mossy Cloud Forest, Lord Howe Island, Australia. Austral Ecology, 40(4), 364-372. doi: 10.1111/aec.12202

Barrett, S., \& Yates, C. J. (2015). Risks to a mountain summit ecosystem with endemic biota in southwestern Australia. Austral Ecology, 40(4), 423-432. doi: 10.1111/aec.12199
Benson, J. S. (2013). Scientific foundations for an IUCN Red List of Ecosystems-Supplementary material (Mock Olive - Wilga Peach Bush - Carissa dry sub-tropical semi-evergreen vine thicket in south eastern Australia). Plos One, 8(5), 104-110.

Bland, L. M., Keith, D. A., Miller, R. M., Murray, N. J., \& Rodríguez, J. P. (2016). Directrices para la aplicación de las Categorías y Criterios de la Lista Roja de Ecosistemas de UICN, Versión 1.0. Gland, Suiza: UICN.

Bland, L. M., Keith, D. A., Miller, R. M., Murray, N. J., \& Rodríguez, J. P. (2017a). Guidelines for the application of IUCN Red List of Ecosystems Categories and Criteria, version 1.1. Gland, Switzerland: IUCN. Ix.

Bland, L. M., Nicholson, E., Miller, R. M., Andrade, A., Carré, A., Etter, A., . . , \& Lindgaard, A. (2019). Impacts of the IUCN Red List of Ecosystems on conservation policy and practice. Conservation letters, 12(5). doi: 10.1111/conl.12666

Bland, L. M., Regan, T. J., Dinh, M. N., Ferrari, R., Keith, D. A., Lester, R., . ., \& Nicholson, E. (2017b). Using multiple lines of evidence to assess the risk of ecosystem collapse. Proc. R. Soc. B, 284(1863), 20170660. doi: 10.1098/rspb.2017.0660

Bland, L. M., Rowland, J. A., Regan, T. J., Keith, D. A., Murray, N. J., Lester, R. E., . . ., \& Nicholson, E. (2018). Developing a standardized definition of ecosystem collapse for risk assessment. Frontiers in Ecology and the Environment, 16(1), 29-36. doi: 10.1002/fee. 1747

Boitani, L., Mace, G. M., \& Rondinini, C. (2015). Challenging the scientific foundations for an IUCN Red List of Ecosystems. Conservation letters, 8(2), 125-131. doi: 10.1111/conl.12111

Bonifacio, R., \& Pisanu, P. (2013). Scientific foundations for an IUCN Red List of Ecosystems-Supplementary material (Karst RisingSpring [KRS] Wetland Community of the South East [SA]). Plos One, 8(5), 198-221.

Bray, D., Duran, E., \& Molina, O. (2012). Beyond harvests in the commons: multi-scale governance and turbulence in indigenous/community conserved areas in Oaxaca, Mexico. International Journal of the Commons, 6(2). doi: 10.18352/ijc.328

Burns, E. L., Lindenmayer, D. B., Stein, J., Blanchard, W., McBurney, L., Blair, D., \& Banks, S. C. (2015). Ecosystem assessment of mountain ash forest in the Central Highlands of Victoria, southeastern Australia. Austral Ecology, 40(4), 386-399. doi: 10.1111/aec.12200

Cardinale, B. J., Duffy, J. E., Gonzalez, A., Hooper, D. U., Perrings, C., Venail, P., . . .. \& Wardle, D. A. (2012). Biodiversity loss and its impact on humanity. Nature, 486(7401), 59. doi: $10.1038 /$ nature11148

Carpenter, S., DeFries, R., Dietz, T., Mooney, H. A., Polasky, S., Reid, W. V., \& Scholes, R. J. (2006). Millennium ecosystem assessment: 
Research needs. science, 314, 257-258. doi: 10.1126/science.1131946

Carré, A., Peguin, M., \& Puolin, B. (2012). Liste Rouge des Écosystèmes de l'UICN. Exercice d'application sur quelques écosystèmes de Zones Humides de France métropolitaine (U.-C. Francais Ed.). France: UICN-Comité Francais.

Crespin, S. J., \& Simonetti, J. A. (2015). Predicting ecosystem collapse: spatial factors that influence risks to tropical ecosystems. Austral Ecology, 40(4), 492-501. doi: 10.1111/aec.12209

Chytrý, M., Hájek, M., Kočí, M., Pešout, P., Roleček, J., Sádlo, J., . . . Douda, J. (2019). Red List of Habitats of the Czech Republic. Ecological Indicators, 106, $105446 . \quad$ doi: 10.1016/j.ecolind.2019.105446

English, V., \& Keith, D. A. (2015). Assessing risks to ecosystems within biodiversity hotspots: a case study from southwestern A ustralia. Austral Ecology, 40(4), 411-422. doi: 10.1111/aec.12177

Essl, F. (2013). Scientific foundations for an IUCN Red List of Ecosystems-Supplementary material (German Tamarisk-Pioneer Vegetation [GTPV], Europe). Plos One, 8(5), 28-35.

Etter, A., Andrade, A., Amaya, P., \& Arévalo, P. (2015). Estado de los ecosistemas colombianos 2014: una aplicación de la metodología de lista roja de ecosistemas UICN: Bogotá, Colombia (V.1 ed., pp. 108).

Etter, A., Andrade, Á., Saavedra, K., Amaya, P., Arévalo, P., Cortés, J., . . ., \& Soler, D. (2017). Lista Roja de Ecosistemas de Colombia (Vers.2.0). In P. U. Javeriana \& C. Internacional-Colombia (Eds.). Bogotá, Colombia: Pontificia Universidad Javeriana y Conservación Internacional-Colombia.

Faber-Langendoen, D., \& Bakowsky, W. (2013). Scientific foundations for an IUCN Red List of Ecosystems-Supplementary material (Great Lakes Alvar). Plos One, 8(5), 140-146.

Ferrer-Paris, J. R., Zager, I., Keith, D. A., Oliveira-Miranda, M. A., Rodríguez, J. P., Josse, C., ..., \& Barrow, E. (2019). An ecosystem risk assessment of temperate and tropical forests of the Americas with an outlook on future conservation strategies. Conservation letters, 12(2), e12623. doi: 10.1111/conl.12623

Hassan, R., Scholes, R. J., \& Ash, N. (2005). Ecosystems and buman wellbeing: current state and trends, vol 1. Findings of the condition and trends working group of the Millennium Ecosystem Assessment (Vol. 1). Washington, DC: Island Press.

Herrera-F, B., Zamora, N., \& Chacón, O. (2015). Lista Roja de los Ecosistemas Terrestres de Costa Rica: Informe final de proyecto (pp. 75). Turrialba, Costa Rica: CATIE.
Holdaway, R., Wiser, S., Richardson, S., \& Arnst, E. (2013). Scientific foundations for an IUCN Red List of Ecosystems-Supplementary material (Granite Gravel Fields \& Sand Plains). Plos One, 8(5), 120125.

Holdaway, R. J., Wiser, S. K., \& Williams, P. A. (2012). Status assessment of New Zealand's naturally uncommon ecosystems. Conservation Biology, 26(4), 619-629. doi: 10.1111/j.1523-1739.2012.01868.x

Iucn-France. (2017). La Liste rouge des écosystèmes en France - Chapitre Mangroves de Mayotte. Paris, France: UICN France.

Janssen, J. A. M., Rodwell, J. S., García-Criado, M., Gubbay, S., Haynes, T., Nieto, A., . . . \& Valachovič, M. (2016). European Red List of Habitats, Part 2. Terrestrial and freshwater habitats. England: Luxembourg: Publications Office of the European Union.

Keith, D. A. (2013a). Scientific foundations for an IUCN Red List of Ecosystems-Supplementary material (Cape Flats Sand Fynbos). Plos One, 8(5), 126-133.

Keith, D. A. (2013b). Scientific foundations for an IUCN Red List of Ecosystems-Supplementary material (Coastal Sandstone Upland Swamps, South-Eastern Australia). Plos One, 8(5), 11-20. doi: 10.1371/journal.pone.0062111.s002

Keith, D. A. (2013c). Scientific foundations for an IUCN Red List of Ecosystems-Supplementary material (Coolibah - Black Box Woodlands, south-eastern Australia). Plos One, 8(5), 95-103.

Keith, D. A., Ba, T., \& Tappan, G. (2013a). Scientific foundations for an IUCN Red List of Ecosystems-Supplementary material (Senegal and Mauritania: Gonakier Forests of Senegal River Floodplain). Plos One, 8(5), 73-82.

Keith, D. A., Rodríguez, J., Rodríguez-Clark, K., Nicholson, E., Aapala, K., Alonso, A., . . ., \& Zambrano-Martínez, S. (2013b). Fundamentos científicos de una Lista Roja de Ecosistemas de UICN. Plos One, 8(5), 1-24. doi: 10.1371/journal.pone.0062111

Kingsford, R. T. (2013). Scientific foundations for an IUCN Red List of Ecosystems-Supplementary material (Swamps, marshes and lakes in the Murray-Darling Basin, Australia). Plos One, 8(5), 36-49.

Kontula, T., \& Raunio, A. (2018). Suomen luontotyyppien uhanalaisuus 2018. Luontotyyppien punainen kirja, CLUS ON, 100.

Mac-Nally, R., Cunningham, S., \& Newell, G. (2013). Scientific foundations for an IUCN Red List of Ecosystems-Supplementary material (The Floodplain Ecosystem of river red gum and black box-south-eastern Australia). Plos One, 8(5), 82-93.

Manzon-Nunes, G., \& Nunes da Cunha, C. (2015). Red List of Ecosystems. Regional Case Studies in Brazil-Pantanal (pp. 2). Brasil: IUCN. 
Marshall, A., Schulte to Bühne, H., Bland, L., \& Pettorelli, N. (2018). Assessing ecosystem collapse risk in ecosystems dominated by foundation species: the case of fringe mangroves. Ecological Indicators, 91, 128-137. doi: 10.1016/j.ecolind.2018.03.076

Metcalfe, D. J., \& Lawson, T. (2015). An International Union for Conservation of Nature risk assessment of coastal lowland rainforests of the Wet Tropics Bioregion, Queensland, Australia. Austral Ecology, 40(4), 373-385. doi: 10.1111/aec.12263

Moat, J., \& Bachman, S. (2013). Scientific foundations for an IUCN Red List of Ecosystems-Supplementary material (Tapia Forest, Madagascar). Plos One, 8(5), 133-138.

Nicholson, E., Collen, B., Barausse, A., Blanchard, J. L., Costelloe, B. T., Sullivan, K. M., . . ., \& Jones, J. P. (2012). Making robust policy decisions using global biodiversity indicators. Plos One, 7(7), e41128. doi: 10.1371/journal.pone.0041128

Nicholson, E., Keith, D. A., \& Wilcove, D. S. (2009). Assessing the threat status of ecological communities. Conservation Biology, 23(2), 259-274. doi: 10.1111/j.1523-1739.2008.01158.x

Nicholson, E., Regan, T. J., Auld, T. D., Burns, E. L., Chisholm, L. A., English, V., . ., \& Leishman, M. R. (2015). Towards consistency, rigour and compatibility of risk assessments for ecosystems and ecological communities. Austral Ecology, 40(4), 347-363. doi: $10.1111 /$ aec. 12148

O'Neill, R. V. (2001). Is it time to bury the ecosystem concept?(with full military honors, of course!). Ecology, 82(12), 3275-3284. doi: 10.1890/0012-9658(2001)082[3275:IITTBT]2.0.CO;2

Oliveira-Miranda, M., Huber, O., Rodríguez, J., Rojas-Suárez, F., De Oliveira-Miranda, R., Zambrano-Martinez, S., \& Giraldo, D. (2010). Riesgo de eliminación de los ecosistemas terrestres de Venezuela. En J. Rodríguez, F. Rojas-Suárez, \& D. Giraldo (Eds.), Libro Rojo de los Ecosistemas Terrestres de Venezuela. (pp. 109-231). Caracas, Venezuela: Provita, Shell Venezuela y Lenovo.

Oliveira-Miranda, M. A., Rodríguez, J. P., \& Zambrano-Martínez, S. (2013). Scientific foundations for an IUCN Red List of Ecosystems-Supplementary material (Tepui shrublands, southern Venezuela). Plos One, 8(5), 111-119.

Pickett, S. T., \& Cadenasso, M. L. (2002). The ecosystem as a multidimensional concept: meaning, model, and metaphor. Ecosystems, 5(1), 1-10. doi: 10.1007/s10021-001-0051-y

Plataforma Intergubernamental Científico-Normativa Sobre Biodiversidad y Servicios de los Ecosistemas [Ipbes] (2019). Summary for policymakers of the global assessment report on biodiversity and ecosystem services of the Intergovernmental Science-Policy Platform on Biodiversity and Ecosystem Services (pp. 56). Bonn, Germany: Ipbes Secretariat.
Pliscoff, P. (2015). Aplicación de los criterios de la Unión Internacional para la Conservación de la Naturaleza (IUCN) para la evaluación de riesgo de los ecosistemas terrestres de Chile. Santiago, Chile: Ministerio del Medio Ambiente.

Poulin, B. (2013). Scientific foundations for an IUCN Red List of Ecosystems-Supplementary material (European Reedbeds). Plos One, 8(5), 64-71.

Redford, K. H. (1992). The empty forest. BioScience, 42(6), 412-422. doi: $10.2307 / 1311860$

Riecken, U., Finck, P., \& Raths, U. (2013). Scientific foundations for an IUCN Red List of Ecosystems-Supplementary material [Raised Bogs of Germany [National Assessment]). Plos One, 8(5), 21-27.

Rodríguez, J. P., Keith, D. A., Rodríguez-Clark, K. M., Murray, N. J., Nicholson, E., Regan, T. J., . . . Wit, P. (2015). A practical guide to the application of the IUCN Red List of Ecosystems criteria. Phil. Trans. R. Soc. B, 370(1662), 20140003.

Rodríguez, J. P., Rodríguez-Clarck, K. M., Baillie, J. E., Ash, N., Benson, J., Boucher, T., . . Jennings, M. (2011). Establishing IUCN red list criteria for threatened ecosystems. Conservation Biology, 25(1), 21-29. doi: 10.1111/j.1523-1739.2010.01598.x

Rodríguez, J. P., Rojas-Suarez, F., \& Giraldo-Hernández, D. (2010). Libro rojo de los ecosistemas terrestres de Venezuela. Caracas: Venezuela: Provita, Shell Venezuela, Lenovo (Venezuela).

Rowland, J. A., Nicholson, E., Murray, N. J., Keith, D. A., Lester, R. E., \& Bland, L. M. (2018). Selecting and applying indicators of ecosystem collapse for risk assessments. Conservation Biology, 32(6), 1233-1245. doi:doi.org/10.1111/cobi.13107

Sato, C. F., \& Lindenmayer, D. B. (2018). Meeting the global ecosystem collapse challenge. Conservation letters, 11(1), e12348. doi: $10.1111 /$ conl. 12348

Serrat, O. (2017). Social network analysis. En O. Serrat (Ed.), Knowledge solutions (pp. 39-43). Singapur: Springer.

Tan, J., Li, A., Lei, G., Bian, J., Chen, G., \& Ma, K. (2017). Preliminary assessment of ecosystem risk based on IUCN criteria in a hierarchy of spatial domains: A case study in Southwestern China. Biological Conservation, 215, 152-161. doi: 10.1016/j.biocon.2017.09.01

Tansley, A. G. (1935). The use and abuse of vegetational concepts and terms. Ecology, 16(3), 284-307. doi:doi.org/10.2307/1930070

Tozer, M. G., Leishman, M. R., \& Auld, T. D. (2015). Ecosystem risk assessment for Cumberland Plain Woodland, New South Wales, Australia. Austral Ecology, 40(4), 400-410. doi: 10.1111/aec.12201

Unión Internacional para la Conservación de la Naturaleza [IUCN] Commission on Ecosystem Management [CEM] (2016). The 
IUCN Red List of Ecosystems. Version 2016-1. Obtenido de https://iucnrle.org/assessments/

Unión Internacional para la Conservación de la Naturaleza [UICN]France (2014). La Liste rouge des écosystèmes en France Habitats forestiers de France métropolitaine - Recueil des études de cas (pp. 89). Paris, France: IUCN Comité Francais.

Unión Internacional para la Conservación de la Naturaleza [UICN]France (2018). La Liste Rouge des Écosystèmes en France: Les Forêts méditerranéennes de France métropolitaine (pp. 28). Paris, France: UICN France.

Valderrábano, M., Benabid, A., Del Barrio, G., Ruiz, A., Sanjuán, M. E., Sainz, H., \& Simón, J. C. (2015). Libro rojo de los ecosistemas forestales de Marruecos. Gland, Suiza y Málaga, España: UICN.

Velazquez, A., Medina-García, C., Durán-Medina, E., Amador, A., \& Gopar-Merino, L. F. (2016). Standardized Hierarcbical Vegetation Classification: Mexican and Global Patterns: Springer.

Wardle, G. M., Greenville, A. C., Frank, A. S., Tischler, M., Emery, N. J., \& Dickman, C. R. (2015). Ecosystem risk assessment of Georgina gidgee woodlands in central Australia. Austral Ecology, 40(4), 444-459. doi: 10.1111/aec.12265
Williams, R. J., Wahren, C.-H., Stott, K. A. J., Camac, J. S., White, M., Burns, E., . . ., \& Hoffmann, A. A. (2015). An I nternational Union for the Conservation of Nature Red List ecosystems risk assessment for alpine snow patch herbfields, South-Eastern Australia. Austral Ecology, 40(4), 433-443. doi: 10.1111/aec.12266

Manuscrito recibido el 25 de febrero de 2020

Aceptado el 18 de febrero de 2021

Publicado el 24 de diciembre de 2021

Este documento se debe citar como:

Masés-García, C. A., Herrera-Fernández, B., \& Briones-Salas, M. (2021). Tendencias en las evaluaciones de riesgo al colapso de ecosistemas terrestres y humedales. Madera y Bosques, 27(3), e2732133. doi: 10.21829/myb.2021.2732133

Madera y Bosques por Instituto de Ecología, A.C. se distribuye bajo una Licencia Creative Commons Atribución-NoComercialCompartirlgual 4.0 Internacional. 